\title{
Numerical Solution of Time-Fractional Diffusion-Wave Equations via Chebyshev Wavelets Collocation Method
}

\author{
Fengying Zhou and Xiaoyong Xu \\ School of Science, East China University of Technology, Nanchang 330013, China \\ Correspondence should be addressed to Fengying Zhou; zhoufengying@ecit.cn
}

Received 11 May 2017; Accepted 6 August 2017; Published 7 September 2017

Academic Editor: Ming Mei

Copyright ( 2017 Fengying Zhou and Xiaoyong Xu. This is an open access article distributed under the Creative Commons Attribution License, which permits unrestricted use, distribution, and reproduction in any medium, provided the original work is properly cited.

\begin{abstract}
The second-kind Chebyshev wavelets collocation method is applied for solving a class of time-fractional diffusion-wave equation. Fractional integral formula of a single Chebyshev wavelet in the Riemann-Liouville sense is derived by means of shifted Chebyshev polynomials of the second kind. Moreover, convergence and accuracy estimation of the second-kind Chebyshev wavelets expansion of two dimensions are given. During the process of establishing the expression of the solution, all the initial and boundary conditions are taken into account automatically, which is very convenient for solving the problem under consideration. Based on the collocation technique, the second-kind Chebyshev wavelets are used to reduce the problem to the solution of a system of linear algebraic equations. Several examples are provided to confirm the reliability and effectiveness of the proposed method.
\end{abstract}

\section{Introduction}

Many phenomena in various fields of the science and engineering can be modeled by fractional differential equations, in which time-fractional diffusion-wave equation is a mathematical model of a wide class of important physical phenomena. It is obtained from the diffusion-wave equation by replacing the second-order time derivative term by a fractional derivative order $1<\alpha \leq 2$. In this paper, our study focuses on the following time-fractional diffusion-wave ([1]) with the Caputo fractional derivative

$$
\begin{aligned}
\frac{\partial^{\alpha} \mu(x, t)}{\partial t^{\alpha}}+\lambda \frac{\partial \mu(x, t)}{\partial t}=\frac{\partial^{2} \mu(x, t)}{\partial x^{2}} & +q(x, t), \\
0 & \leq x \leq 1,0 \leq t \leq 1,
\end{aligned}
$$

with the initial condition

$$
\begin{aligned}
\mu(x, 0) & =f_{0}(x), \\
\frac{\partial \mu(x, 0)}{\partial t} & =f_{1}(x),
\end{aligned}
$$

$$
0 \leq x \leq 1
$$

and the boundary conditions

$$
\begin{aligned}
& \mu(0, t)=g_{0}(t), \\
& \mu(1, t)=g_{1}(t), \\
& 0 \leq t \leq 1,
\end{aligned}
$$

where $f_{0}(x), f_{1}(x), g_{0}(t), g_{1}(t)$ are given functions with second-order continuous derivatives and $\lambda>0$ is a constant and $q(x, t)$ is a given known function.

It is noted that most fractional diffusion-wave equations do not have closed form solutions. Many researchers have proposed various methods to solve the time-fractional diffusion-wave equations from the perspective of analytical solution and numerical solution. The method of separation of variables in [1], Sumudu transform method in [2], and decomposition method in [3] were used to construct analytical approximate solutions of fractional diffusion-wave equations, respectively. Finite difference schemes in [47] were widely used to solve the numerical solutions of the fractional diffusion-wave equations. The authors of [8] employed radial point interpolation method for solving the fractional diffusion-wave equations. B-spline collocation method was proposed to solve the fractional diffusion-wave 
equations in [9]. In [10, 11], Sinc-finite difference method and Sinc-Chebyshev method were employed for solving the fractional diffusion-wave equations respectively. Recently methods based on operational matrix of Jacobi and Chebyshev polynomials were proposed to deal with the fractional diffusion-wave equations ([12-14]). In [15], the authors applied fractional order Legendre functions method depending on the choices of two parameters to solve the fractional diffusion-wave equations. Two-dimensional Bernoulli wavelets with satisfier function in the Ritz-Galerkin method were proposed for the time-fractional diffusion-wave equation in [16]. The author of [17] proposed a numerical method based on the Legendre wavelets with their operational matrix of fractional integral to solve the time-fractional diffusionwave equations.

Since fractional derivative is a nonlocal operator, it is natural to consider a global scheme such as the collocation method for its numerical solution. Spectral methods are widely used in seeking numerical solutions of fractional order differential equations, due to their excellent error properties and exponential rates of convergence for smooth problems. Collocation methods, one of the three most common spectral schemes, have been applied successfully to numerical simulations of many problems in science and engineering.

Wavelets, as another basis set and very well-localized functions, are considerably useful for solving differential and integral equations. Particularly, orthogonal wavelets are widely used in approximating numerical solutions of various types of fractional order differential equations in the relevant literatures; see [18-22]. Among them, the second-kind Chebyshev wavelets have gained much attention due to their useful properties ([23-26]) and can handle different types of differential problems. It is observed that most papers using these wavelets methods to approximate numerical solutions of fractional order differential equations are based on the operational matrix of fractional integral or fractional derivatives. It is inevitable to produce approximation error during the process of constructing the operational matrix. Regarding this point, analysis in [27] shows some disadvantages of using the operational matrix of Legendre and Chebyshev wavelets.

Inspired and motivated by the work mentioned above, our main purpose of this paper is to extend the second-kind Chebyshev wavelets for solving time-fractional diffusionwave equations (1)-(3) and to show that it is not necessary to establish the operational matrix of fractional integrals and fractional derivatives when applying wavelets to solve various types of fractional partial differential equations. To reduce the approximation error at most during the calculation process, fractional integral formula of a single Chebyshev wavelet in the Riemann-Liouville sense is derived by means of the shifted Chebyshev polynomials of the second kind. By utilizing the collocation method and some properties of the second-kind Chebyshev wavelets, the problem under consideration is reduced to the solution to a system of linear algebraic equations. The proposed method is very convenient for solving such problems, since the initial and boundary conditions are taken into account automatically.
The rest of the paper is organized as follows. Section 2 describes some necessary definitions and preliminaries of calculus. Section 3 gives the convergence and accuracy estimation of the second-kind Chebyshev wavelets expansion of two-dimension. Section 4 is devoted to deriving the fractional integral formula of a single Chebyshev wavelet in the Riemann-Liouville sense by means of shifted Chebyshev polynomials of the second kind. The proposed method is described for solving time-fractional diffusion-wave equations in Section 5. In Section 6, numerical results of some test problems are presented. Finally, a brief conclusion is given in Section 7.

\section{Definitions and Preliminaries}

In this section, we present some necessary definitions and preliminaries of the fractional calculus theory which will be used later. The widely used definitions of fractional integral and fractional derivative are the Riemann-Liouville definition and the Caputo definition, respectively.

Definition 1. A real function $f(x), x>0$, is said to be in the space $C_{\sigma}, \sigma \in \mathbb{R}$, if there is a real number $\rho$ with $\rho>\sigma$ such that $f(x)=x^{\rho} f_{0}(x)$, where $f_{0}(x) \in C[0, \infty)$, and $f(x) \in C_{\sigma}^{n}$ if $f^{(n)}(x) \in C_{\sigma}, n \in \mathbb{N}$.

Definition 2 (see [28]). The Riemann-Liouville fractional integral operator $I^{\alpha}$ of order $\alpha(\alpha \geq 0)$ for a function $f(x) \epsilon$ $C_{\sigma}(\sigma \geq-1)$ is defined as

$$
I^{\alpha} f(x)= \begin{cases}f(x), & \alpha=0, \\ \frac{1}{\Gamma(\alpha)} \int_{0}^{x}(x-t)^{\alpha-1} f(t) d t, & \alpha>0 .\end{cases}
$$

Definition 3 (see [28]). The Caputo fractional derivative operator $D^{\alpha}$ of order $\alpha(\alpha \geq 0)$ for a function $f(x) \in C_{1}^{n}$ is defined as

$$
\begin{aligned}
& D^{\alpha} f(x) \\
& \quad= \begin{cases}f^{(n)}(x), & \alpha=n \in \mathbb{N}, \\
\frac{1}{\Gamma(n-\alpha)} \int_{0}^{x} \frac{f^{(n)}(t)}{(x-t)^{\alpha+1-n}} d t, & n-1<\alpha<n .\end{cases}
\end{aligned}
$$

Some important properties of the operators $I^{\alpha}$ and $D^{\alpha}$ are needed in this paper; we only mention the following properties:

(1) $I^{\alpha_{1}} I^{\alpha_{2}} f(x)=I^{\alpha_{1}+\alpha_{2}} f(x)$ for $\alpha_{1}, \alpha_{2}>0$.

(2) $D^{\alpha} I^{\alpha} f(x)=f(x), D^{\beta} I^{\alpha} f(x)=I^{\alpha-\beta} f(x), \alpha>\beta$.

(3) $I^{\alpha} D^{\alpha} f(x)=f(x)-\sum_{k=0}^{[\alpha]-1} f^{(k)}\left(0^{+}\right)\left(x^{k} / k !\right), x>0$, where the ceiling function $\lceil\alpha\rceil$ denotes the integer smaller than or equal to $\alpha$ and $\mathbb{N}_{0}=\{0,1,2, \ldots\}$. One can see more details about fractional calculus in [28]. 


\section{The Second-Kind Chebyshev Wavelets and Their Properties}

The second-kind Chebyshev wavelets $\psi_{n, m}(t)=\psi(k, n, m, t)$ have four arguments: $k$ can assume any positive integer, $n=1$, $2,3, \ldots, 2^{k-1}, m$, is the degree of the second-kind Chebyshev polynomials and $t$ is the normalized time. They are defined on the interval $[0,1)$ as

$$
\begin{aligned}
& \psi_{n, m}(t) \\
& \quad= \begin{cases}2^{k / 2} \widetilde{U}_{m}\left(2^{k} t-2 n+1\right), & \frac{n-1}{2^{k-1}} \leq t<\frac{n}{2^{k-1}}, \\
0, & \text { otherwise, }\end{cases}
\end{aligned}
$$

where

$$
\widetilde{U}_{m}(t)=\sqrt{\frac{2}{\pi}} U_{m}(t)
$$

$m=0,1,2, \ldots$. Here $U_{m}(t)$ are the second-kind Chebyshev polynomials of degree $m$ which are orthogonal with respect to the weight function $\omega(t)=\sqrt{1-t^{2}}$ on the interval $[-1,1]$ and satisfy the following recursive formula:

$$
\begin{aligned}
U_{0}(t) & =1, \\
U_{1}(t) & =2 t \\
U_{m+1}(t) & =2 t U_{m}(t)-U_{m-1}(t), \quad m=1,2,3, \ldots .
\end{aligned}
$$

Note that when dealing with the second-kind Chebyshev wavelets the weight function has to be dilated and translated as

$$
\omega_{n}(t)=\omega\left(2^{k} t-2 n+1\right)
$$

A function $f(x) \in L^{2}(\mathbb{R})$ defined over $[0,1)$ may be expanded by the second-kind Chebyshev wavelets as

$$
f(x)=\sum_{n=1}^{\infty} \sum_{m=0}^{\infty} c_{n, m} \psi_{n, m}(x)
$$

where

$$
\begin{aligned}
c_{n, m} & =\left\langle f(x), \psi_{n, m}(x)\right\rangle_{L_{\omega}^{2}[0,1)} \\
& =\int_{0}^{1} f(x) \psi_{n, m}(x) \omega_{n}(x) d x,
\end{aligned}
$$

in which $\langle\cdot, \cdot\rangle_{L_{\omega}^{2}[0,1)}$ denotes the inner product in $L_{\omega}^{2}[0,1)$. If the infinite series in (10) is truncated, then it can be written as

$$
f(x) \cong \sum_{n=1}^{2^{k-1}} \sum_{m=0}^{M-1} c_{n, m} \psi_{n, m}(x)=\mathbf{C}^{T} \Psi(x),
$$

where $\mathbf{C}$ and $\Psi(x)$ are $2^{k-1} M \times 1$ matrices given by

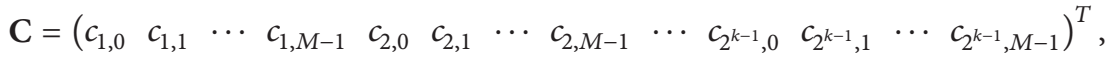

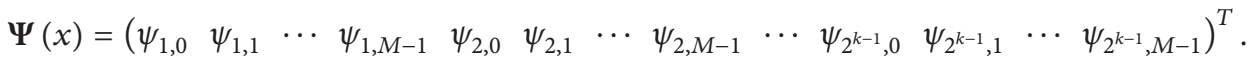

Observe that $\left\{\psi_{n, m}(x) \psi_{n^{\prime}, m^{\prime}}(y): n, n^{\prime}=1,2,3, \ldots, 2^{k-1}\right.$, $\left.m, m^{\prime}=0,1,2, \ldots\right\}$ is an orthonormal set over $[0,1) \times[0,1)$. A function $\mu(x, y) \in L^{2}\left(\mathbb{R}^{2}\right)$ defined over $[0,1) \times[0,1)$ may be expanded as

$$
\mu(x, y)=\sum_{n=1}^{2^{k-1}} \sum_{n^{\prime}=1}^{2^{k-1}} \sum_{m=0}^{\infty} \sum_{m^{\prime}=0}^{\infty} d_{n, n^{\prime}, m, m^{\prime}} \psi_{n, m}(x) \psi_{n^{\prime}, m^{\prime}}(y),
$$

where

$$
\begin{aligned}
& d_{n, n^{\prime}, m, m^{\prime}}=\left\langle\mu(x, y), \psi_{n, m}(x) \psi_{n^{\prime}, m^{\prime}}(y)\right\rangle_{L_{\omega}^{2}([0,1) \times[0,1))} \\
& \quad=\int_{0}^{1} \int_{0}^{1} \mu(x, y) \psi_{n, m}(x) \psi_{n^{\prime}, m^{\prime}}(y) \omega_{n}(x) \\
& \cdot \omega_{n^{\prime}}(y) d x d y .
\end{aligned}
$$

If the infinite series in (14) is truncated, then (14) can be written as

$$
\begin{aligned}
& \mu(x, y) \cong \sum_{n=1}^{2^{k-1}} \sum_{n^{\prime}=1}^{2^{k-1}} \sum_{m=0}^{M-1} \sum_{m^{\prime}=0}^{M-1} d_{n, n^{\prime}, m, m^{\prime}} \psi_{n, m}(x) \psi_{n^{\prime}, m^{\prime}}(y) \\
& =\sum_{n=1}^{2^{k-1}} \sum_{m=0}^{M-1} \psi_{n, m}(x)\left(\sum_{n^{\prime}=1}^{2^{k-1}} \sum_{m^{\prime}=0}^{M-1} d_{n, n^{\prime}, m, m^{\prime}} \psi_{n^{\prime}, m^{\prime}}(y)\right) \\
& =\sum_{n=1} \sum_{m=0}^{M-1} \psi_{n, m}(x) \mathbf{D}_{n, m}^{T} \Psi(y),
\end{aligned}
$$

where $\mathbf{D}_{n, m}$ and $\boldsymbol{\Psi}(y)$ are $2^{k-1} M \times 1$ matrices given by

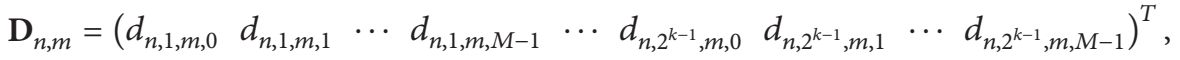

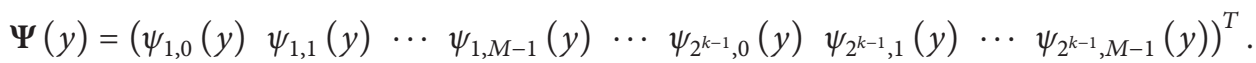


Write $\Psi_{n}(x)=\left(\psi_{n, 0}(x) \quad \psi_{n, 1}(x) \cdots \psi_{n, M-1}(x)\right)$ and $\mathbf{D}_{n}=$ $\left(\begin{array}{llll}\mathbf{D}_{n, 0} & \mathbf{D}_{n, 1} & \cdots & \mathbf{D}_{n, M-1}\end{array}\right)^{T}, n=1,2,3, \ldots, 2^{k-1}$; then $\mu(x, y)$ can be written as

$$
\begin{aligned}
\mu(x, y) & \cong \sum_{n=1}^{2^{k-1}} \sum_{m=0}^{M-1} \psi_{n, m}(x) \mathbf{D}_{n, m}^{T} \Psi(y) \\
& =\sum_{n=1}^{2^{k-1}} \Psi_{n}(x) \mathbf{D}_{n} \Psi(y) \\
& =\left(\begin{array}{lll}
\Psi_{1}(x) & \cdots & \Psi_{2^{k-1}}(x)
\end{array}\right)\left(\begin{array}{c}
\mathbf{D}_{1} \\
\mathbf{D}_{2} \\
\vdots \\
\mathbf{D}_{2^{k-1}}
\end{array}\right) \Psi(y) \\
& =\Psi^{T}(x) \mathbf{D} \Psi(y),
\end{aligned}
$$

where $\mathbf{D}$ is a $2^{k-1} M \times 2^{k-1} M$ matrix given by $\mathbf{D}=\left(\begin{array}{c}\mathbf{D}_{1} \\ \mathbf{D}_{2} \\ \vdots \\ \mathbf{D}_{2^{k-1}}\end{array}\right)$.

The following theorems give the convergence and accuracy estimation of the second-kind Chebyshev wavelets expansion.

Theorem 4 (see [29]). Let $f(x)$ be a second-order derivative square-integrable function defined on $[0,1)$ with bounded second-order derivative; say $\left|f^{\prime \prime}(x)\right| \leq B$ for some constant $B$; then

(i) $f(x)$ can be expanded as an infinite sum of the secondkind Chebyshev wavelets and the series converges to $f(x)$ uniformly; that is,

$$
f(x)=\sum_{n=1}^{\infty} \sum_{m=0}^{\infty} c_{n, m} \psi_{n, m}(x)
$$

where $c_{n, m}=\left\langle f(x), \psi_{n, m}(x)\right\rangle_{L_{\omega}^{2}[0,1)}$; (ii)

$$
\sigma_{f, k, M}<\frac{\sqrt{\pi} B}{2^{3}}\left(\sum_{n=2^{k-1}+1}^{\infty} \frac{1}{n^{5}} \sum_{m=M}^{\infty} \frac{1}{(m-1)^{4}}\right)^{1 / 2},
$$

where $\quad \sigma_{f, k, M} \quad\left(\int_{0}^{1} \mid f(x)\right.$ $\left.\left.\sum_{n=1}^{2^{k-1}} \sum_{m=0}^{M-1} c_{n, m} \psi_{n, m}(x)\right|^{2} \omega_{n}(x) d x\right)^{1 / 2}$.

Theorem 5. Supposing that $\mu(x, y) \in L^{2}\left(\mathbb{R}^{2}\right)$ is a continuous function defined on $[0,1) \times[0,1), \partial^{2} \mu(x, y) / \partial x^{2}, \partial^{2} \mu(x, y) /$ $\partial y^{2}$, and $\partial^{4} \mu(x, y) / \partial x^{2} \partial y^{2}$ are bounded with some positive constant $B$. Then, for any positive integer $k$,

(i) the series

$$
\sum_{n=1}^{2^{k-1}} \sum_{n^{\prime}=1}^{2^{k-1}} \sum_{m=0}^{\infty} \sum_{m^{\prime}=0}^{\infty} d_{n, m, n^{\prime}, m^{\prime}} \psi_{n, m}(x) \psi_{n^{\prime}, m^{\prime}}(y)
$$

converges to $\mu(x, y)$ uniformly in $L^{2}\left(\mathbb{R}^{2}\right)$; that is,

$$
\begin{aligned}
& \mu(x, y) \\
& =\sum_{n=1}^{2^{k-1}} \sum_{n^{\prime}=1}^{2^{k-1}} \sum_{m=0}^{\infty} \sum_{m^{\prime}=0}^{\infty} d_{n, m, n^{\prime}, m^{\prime}} \psi_{n, m}(x) \psi_{n^{\prime}, m^{\prime}}(y),
\end{aligned}
$$

where $d_{n, m, n^{\prime}, m^{\prime}}=\left\langle\mu(x, y), \psi_{n, m}(x) \psi_{n^{\prime}, m^{\prime}}(y)\right\rangle_{L_{\omega}^{2}([0,1) \times[0,1))}$;

(ii)

$$
\begin{aligned}
& \sigma_{\mu, k, M}^{2}<\frac{(B \pi)^{2}}{2^{2}} \sum_{n=1}^{2^{k-1}} \frac{1}{n^{3}} \sum_{n^{\prime}=1}^{2^{k-1}} \frac{1}{n^{\prime 3}} \sum_{m=M}^{\infty} \frac{1}{m^{2}(m-1)^{2}} \\
& +\frac{(B \pi)^{2}}{2^{15}} \\
& \quad \cdot \sum_{n=1}^{2^{k-1}} \frac{1}{n^{5}} \sum_{n^{\prime}=1}^{2^{k-1}} \frac{1}{n^{\prime 5}} \sum_{m=2}^{\infty} \frac{1}{m^{2}(m-1)^{2}} \sum_{m^{\prime}=M}^{\infty} \frac{1}{m^{\prime 2}\left(m^{\prime}-1\right)^{2}}
\end{aligned}
$$

where

$$
\sigma_{\mu, k, M}=\left(\int_{0}^{1} \int_{0}^{1}\left|\mu(x, y)-\sum_{n=1}^{2^{k-1}} \sum_{n^{\prime}=1}^{2^{k-1}} \sum_{m=0}^{M-1} \sum_{m^{\prime}=0}^{M-1} d_{n, n^{\prime}, m, m^{\prime}} \psi_{n, m}(x) \psi_{n^{\prime}, m^{\prime}}(y)\right|^{2} \omega_{n}(x) \omega_{n^{\prime}}(y) d x d y\right)^{1 / 2} .
$$

Proof. (i)

$$
\begin{aligned}
& d_{n, m, n^{\prime}, m^{\prime}}=\left\langle\mu(x, y), \psi_{n, m}(x) \psi_{n^{\prime}, m^{\prime}}(y)\right\rangle_{L_{\omega}^{2}([0,1) \times[0,1))} \\
& =\int_{0}^{1} \int_{0}^{1} \mu(x, y) \psi_{n, m}(x) \psi_{n^{\prime}, m^{\prime}}(y) \omega_{n}(x) \\
& \cdot \omega_{n^{\prime}}(y) d x d y=\int_{0}^{1} \psi_{n^{\prime}, m^{\prime}}(y) \omega_{n^{\prime}}(y) \\
& \cdot\left(\int_{0}^{1} \mu(x, y) \psi_{n, m}(x) \omega_{n}(x) d x\right) d y
\end{aligned}
$$

$$
=\int_{0}^{1} \psi_{n^{\prime}, m^{\prime}}(y) \omega_{n^{\prime}}(y) A_{n, m}(y) d y
$$

where $A_{n, m}(y)=\int_{0}^{1} \mu(x, y) \psi_{n, m}(x) \omega_{n}(x) d x$. Similar to the proof of Theorem 1 in [29], we get, for $m>1$,

$$
\begin{aligned}
& A_{n, m}(y)=\frac{2^{-5 k / 2}}{2 \sqrt{2 \pi}} \frac{1}{m(m-1)(m+1)} \\
& \cdot \int_{0}^{\pi} \frac{\partial^{2} \mu\left((\cos \theta+2 n-1) / 2^{k}, y\right)}{\partial \theta^{2}} \tau_{m}(\theta) d \theta,
\end{aligned}
$$


where $\tau_{m}(\theta)=(m+1) \sin (m-1) \theta \sin \theta-(m-1) \sin (m+$ 1) $\theta \sin \theta$. So, for $m>1$ and $m^{\prime}>1$,

$$
\begin{aligned}
& d_{n, m, n^{\prime}, m^{\prime}}=\int_{0}^{1} \psi_{n^{\prime}, m^{\prime}}(y) \omega_{n^{\prime}}(y) A_{n, m}(y) d y=\frac{2^{-5 k / 2}}{2 \sqrt{2 \pi}} \frac{1}{m(m-1)(m+1)} \\
& \cdot \int_{0}^{\pi} \tau_{m}(\theta) \int_{0}^{1} \frac{\partial^{2} \mu\left((\cos \theta+2 n-1) / 2^{k}, y\right)}{\partial \theta^{2}} \cdot \psi_{n^{\prime}, m^{\prime}}(y) \omega_{n^{\prime}}(y) d y d \theta=\frac{2^{-5 k / 2}}{2 \sqrt{2 \pi}} \frac{1}{m(m-1)(m+1)} \\
& \cdot \int_{0}^{\pi} \tau_{m}(\theta) \cdot \frac{2^{-5 k / 2}}{2 \sqrt{2 \pi}} \frac{1}{m^{\prime}\left(m^{\prime}-1\right)\left(m^{\prime}+1\right)} \cdot \int_{0}^{\pi} \frac{\partial^{4} \mu\left((\cos \theta+2 n-1) / 2^{k},\left(\cos \eta+2 n^{\prime}-1\right) / 2^{k}\right)}{\partial \theta^{2} \partial \eta^{2}} \tau_{m^{\prime}}(\eta) d \eta d \theta=\frac{2^{-5 k}}{2^{3} \pi} \\
& \cdot \frac{1}{m(m-1)(m+1)} \frac{m^{\prime}\left(m^{\prime}-1\right)\left(m^{\prime}+1\right)}{\partial \theta^{2} \partial \eta^{2}} \\
& \cdot \int_{0}^{\pi} \int_{0}^{\pi} \frac{\partial^{4} \mu\left((\cos \theta+2 n-1) / 2^{k},\left(\cos \eta+2 n^{\prime}-1\right) / 2^{k}\right)}{\tau_{m}(\theta) \tau_{m^{\prime}}(\eta) d \theta d \eta .}
\end{aligned}
$$

Note that, for $m>1$,

$$
\begin{aligned}
& \left(\int_{0}^{\pi}\left|\tau_{m}(\theta)\right| d \theta\right)^{2} \leq \pi \int_{0}^{\pi}\left|\tau_{m}(\theta)\right|^{2} d \theta \\
& \quad \leq \pi \int_{0}^{\pi} \mid(m+1) \sin (m-1) \theta \\
& \quad-\left.(m-1) \sin (m+1) \theta\right|^{2} d \theta \\
& \quad \leq \pi\left(\int_{0}^{\pi}(m+1)^{2} \sin ^{2}(m-1) \theta d \theta\right. \\
& \left.\quad+\int_{0}^{\pi}(m-1)^{2} \sin ^{2}(m+1) \theta d \theta\right)=\frac{\pi^{2}}{2}\left((m+1)^{2}\right. \\
& \left.\quad+(m-1)^{2}\right) \leq \pi^{2}(m+1)^{2} .
\end{aligned}
$$

$$
\frac{1}{m^{\prime}\left(m^{\prime}-1\right)}
$$

since $n \leq 2^{k-1}$ and $n^{\prime} \leq 2^{k-1}$.

For $m>1$ and $m^{\prime}=0,1$,

$$
\begin{aligned}
& d_{n, m, n^{\prime}, m^{\prime}}=\int_{0}^{1} \psi_{n^{\prime}, m^{\prime}}(y) \omega_{n^{\prime}}(y) A_{n, m}(y) d y=\frac{2^{-5 k / 2}}{2 \sqrt{2 \pi}} \\
& \cdot \frac{1}{m(m-1)(m+1)} \int_{0}^{\pi} \tau_{m}(\theta) \\
& \cdot \int_{0}^{1} \frac{\partial^{2} \mu\left((\cos \theta+2 n-1) / 2^{k}, y\right)}{\partial \theta^{2}} \cdot \psi_{n^{\prime}, m^{\prime}}(y)
\end{aligned}
$$

$$
\begin{aligned}
& \cdot \omega_{n^{\prime}}(y) d y d \theta=\frac{2^{-5 k / 2}}{2 \sqrt{2 \pi}} \frac{1}{m(m-1)(m+1)} \\
& \cdot \int_{0}^{\pi} \tau_{m}(\theta) \int_{\left(n^{\prime}-1\right) / 2^{k-1}}^{n^{\prime} / 2^{k-1}} \frac{\partial^{2} \mu\left((\cos \theta+2 n-1) / 2^{k}, y\right)}{\partial \theta^{2}} \\
& \cdot 2^{k / 2} \sqrt{\frac{2}{\pi}} U_{m^{\prime}}\left(2^{k} y-2 n^{\prime}+1\right) \omega\left(2^{k} y-2 n^{\prime}+1\right) d y d \theta \\
& =\frac{2^{-3 k}}{2 \pi} \frac{1}{m(m-1)(m+1)} \int_{0}^{\pi} \tau_{m}(\theta) \\
& \cdot \int_{0}^{\pi} \frac{\partial^{2} \mu\left((\cos \theta+2 n-1) / 2^{k},\left(\cos \eta+2 n^{\prime}-1\right) / 2^{k}\right)}{\partial \theta^{2}} \\
& \cdot \sin \left(m^{\prime}+1\right) \eta \sin \eta d \eta d \theta
\end{aligned}
$$

where we use the variable substitution $2^{k} y-2 n^{\prime}+1=\cos \eta$ and the definition of the second-kind Chebyshev polynomials. Therefore, for $m>1$ and $m^{\prime}=0,1$, we obtain

$$
\left|d_{n, m, n^{\prime}, m^{\prime}}\right| \leq \frac{B \pi}{2^{4}} \cdot \frac{1}{n^{3 / 2}} \cdot \frac{1}{n^{\prime(3 / 2)}} \cdot \frac{1}{m(m-1)}
$$

by $n \leq 2^{k-1}$ and $n^{\prime} \leq 2^{k-1}$. Similarly, for $m^{\prime}>1$ and $m=0,1$, we also have

$$
\left|d_{n, m, n^{\prime}, m^{\prime}}\right| \leq \frac{B \pi}{2^{4}} \cdot \frac{1}{n^{3 / 2}} \cdot \frac{1}{n^{\prime(3 / 2)}} \cdot \frac{1}{m^{\prime}\left(m^{\prime}-1\right)} .
$$

Relations (29), (31), and (32) show that the series

$$
\sum_{n=1}^{2^{k-1}} \sum_{n^{\prime}=1}^{2^{k-1}} \sum_{m=0}^{\infty} \sum_{m^{\prime}=0}^{\infty} d_{n, m, n^{\prime}, m^{\prime}} \psi_{n, m}(x) \psi_{n^{\prime}, m^{\prime}}(y)
$$

converges to $\mu(x, y)$ uniformly in $L^{2}\left(\mathbb{R}^{2}\right)$. 
(ii)

$$
\begin{aligned}
& \sigma_{\mu, k, M}^{2}=\int_{0}^{1} \int_{0}^{1}\left|\mu(x, y)-\sum_{n=1}^{2^{k-1}} \sum_{n^{\prime}=1}^{2^{k-1}} \sum_{m=0}^{M-1} \sum_{m^{\prime}=0}^{M-1} d_{n, n^{\prime}, m, m^{\prime}} \psi_{n, m}(x) \psi_{n^{\prime}, m^{\prime}}(y)\right|^{2} \cdot \omega_{n}(x) \omega_{n^{\prime}}(y) d x d y \\
& =\int_{0}^{1} \int_{0}^{1}\left|\sum_{n=1}^{2^{k-1}} \sum_{n^{\prime}=1}^{2^{k-1}}\left(\sum_{m^{\prime}=0}^{M-1} \sum_{m=M}^{\infty}+\sum_{m=0}^{M-1} \sum_{m^{\prime}=M}^{\infty}+\sum_{m^{\prime}=M}^{\infty} \sum_{m=M}^{\infty}\right) d_{n, n^{\prime}, m, m^{\prime}} \cdot \psi_{n, m}(x) \psi_{n^{\prime}, m^{\prime}}(y)\right|^{2} \omega_{n}(x) \omega_{n^{\prime}}(y) d x d y .
\end{aligned}
$$

According to the orthonormality of $\psi_{n, m}(x) \psi_{n^{\prime}, m^{\prime}}(y)$, we have

$$
\begin{gathered}
\sigma_{\mu, k, M}^{2}=\sum_{n=1}^{2^{k-1}} \sum_{n^{\prime}=1}^{2^{k-1}}\left(\sum_{m^{\prime}=0}^{M-1} \sum_{m=M}^{\infty}+\sum_{m=0}^{M-1} \sum_{m^{\prime}=M}^{\infty}+\sum_{m^{\prime}=M}^{\infty} \sum_{m=M}^{\infty}\right) \\
\cdot\left|d_{n, n^{\prime}, m, m^{\prime}}\right|^{2}=\sum_{n=1}^{2^{k-1}} \sum_{n^{\prime}=1}^{2^{k-1}}\left(\sum_{m^{\prime}=0}^{1} \sum_{m=M}^{\infty}+\sum_{m^{\prime}=2}^{M-1} \sum_{m=M}^{\infty}\right. \\
\left.+\sum_{m=0}^{1} \sum_{m^{\prime}=M}^{\infty}+\sum_{m=2}^{M-1} \sum_{m^{\prime}=M}^{\infty}+\sum_{m^{\prime}=M}^{\infty} \sum_{m=M}^{\infty}\right) \cdot\left|d_{n, n^{\prime}, m, m^{\prime}}\right|^{2} .
\end{gathered}
$$

By relations (29), (31), and (32), it gives

$$
\begin{aligned}
& \sigma_{\mu, k, M}^{2} \leq \sum_{n=1}^{2^{k-1}} \sum_{n^{\prime}=1}^{2^{k-1}}\left(\sum_{m^{\prime}=0}^{1} \sum_{m=M}^{\infty} \frac{(B \pi)^{2}}{2^{8}} \frac{1}{n^{3}} \frac{1}{n^{\prime 3}} \frac{1}{m^{2}(m-1)^{2}}\right. \\
& +\sum_{m^{\prime}=2}^{M-1} \sum_{m=M}^{\infty} \frac{(B \pi)^{2}}{2^{16}} \frac{1}{n^{5}} \frac{1}{n^{\prime 5}} \frac{1}{m^{2}(m-1)^{2}} \frac{1}{m^{\prime 2}\left(m^{\prime}-1\right)^{2}} \\
& +\sum_{m=0}^{1} \sum_{m^{\prime}=M}^{\infty} \frac{(B \pi)^{2}}{2^{8}} \frac{1}{n^{3}} \frac{1}{n^{\prime 3}} \frac{1}{m^{\prime 2}\left(m^{\prime}-1\right)^{2}} \\
& +\sum_{m=2}^{M-1} \sum_{m^{\prime}=M}^{\infty} \frac{(B \pi)^{2}}{2^{16}} \frac{1}{n^{5}} \frac{1}{n^{\prime 5}} \frac{1}{m^{2}(m-1)^{2}} \frac{1}{m^{\prime 2}\left(m^{\prime}-1\right)^{2}} \\
& \left.+\sum_{m=M}^{\infty} \sum_{m^{\prime}=M}^{\infty} \frac{(B \pi)^{2}}{2^{16}} \frac{1}{n^{5}} \frac{1}{n^{\prime 5}} \frac{1}{m^{2}(m-1)^{2}} \frac{1}{m^{\prime 2}\left(m^{\prime}-1\right)^{2}}\right)
\end{aligned}
$$

$$
\begin{aligned}
& =\sum_{n=1}^{2^{k-1}} \sum_{n^{\prime}=1}^{2^{k-1}}\left(\sum_{m=M}^{\infty} \frac{(B \pi)^{2}}{2^{6}} \frac{1}{n^{3}} \frac{1}{n^{\prime 3}} \frac{1}{m^{2}(m-1)^{2}}\right. \\
& +\sum_{m=2}^{M-1} \sum_{m^{\prime}=M}^{\infty} \frac{(B \pi)^{2}}{2^{15}} \frac{1}{n^{5}} \frac{1}{n^{\prime 5}} \frac{1}{m^{2}(m-1)^{2}} \frac{1}{m^{\prime 2}\left(m^{\prime}-1\right)^{2}} \\
& \left.+\sum_{m=M}^{\infty} \sum_{m^{\prime}=M}^{\infty} \frac{(B \pi)^{2}}{2^{16}} \frac{1}{n^{5}} \frac{1}{n^{\prime 5}} \frac{1}{m^{2}(m-1)^{2}} \frac{1}{m^{\prime 2}\left(m^{\prime}-1\right)^{2}}\right) \\
& <\frac{(B \pi)^{2}}{2^{6}} \sum_{n=1}^{2^{k-1}} \frac{1}{n^{3}} \sum_{n^{\prime}=1}^{2^{k-1}} \frac{1}{n^{\prime 3}} \sum_{m=M}^{\infty} \frac{1}{m^{2}(m-1)^{2}}+\frac{(B \pi)^{2}}{2^{15}} \\
& \cdot \sum_{n=1}^{2^{k-1}} \frac{1}{n^{5}} \sum_{n^{\prime}=1}^{2^{k-1}} \frac{1}{n^{\prime 5}} \sum_{m=2}^{\infty} \frac{1}{m^{2}(m-1)^{2}} \sum_{m^{\prime}=M}^{\infty} \frac{1}{m^{\prime 2}\left(m^{\prime}-1\right)^{2}} .
\end{aligned}
$$

The proof is completed.

\section{The Fractional Integral of a Single Second- Kind Chebyshev Wavelet}

In this section, fractional integral formula of a single Chebyshev wavelet in the Riemann-Liouville sense is derived by means of the shifted second-kind Chebyshev polynomials $U_{m}^{*}$, which plays an important role in dealing with the timefractional diffusion-wave equations.

Theorem 6. The fractional integral of a Chebyshev wavelet defined on the interval $[0,1]$ with compact support $[(n-$ 1) $/ 2^{k-1}, n / 2^{k-1}$ ] is given by

$$
I^{\alpha} \psi_{n, m}(x)= \begin{cases}0, & x<\frac{n-1}{2^{k-1}}, \\ \frac{1}{\Gamma(\alpha)} 2^{k / 2} \sqrt{\frac{2}{\pi}} \sum_{r=0}^{m} \sum_{i=r}^{m} \sum_{j=0}^{r} T_{i, i-r}^{m, n, k} \frac{(-1)^{j}}{j+\alpha} C_{r}^{j} x^{r-j}\left(x-\frac{n-1}{2^{k-1}}\right)^{j+\alpha}, & \frac{n-1}{2^{k-1}} \leq x \leq \frac{n}{2^{k-1}}, \\ \frac{1}{\Gamma(\alpha)} 2^{k / 2} \sqrt{\frac{2}{\pi}} \sum_{r=0}^{m} \sum_{i=r}^{m} \sum_{j=0}^{r} T_{i, i-r}^{m, n} \frac{(-1)^{j}}{j+\alpha} C_{r}^{j} x^{r-j}\left[\left(x-\frac{n-1}{2^{k-1}}\right)^{j+\alpha}-\left(x-\frac{n}{2^{k-1}}\right)^{j+\alpha}\right], & x>\frac{n}{2^{k-1}},\end{cases}
$$


where $T_{i, i-r}^{m, n, k}=(-1)^{m-r} 2^{2 i} 2^{r(k-1)}(n-1)^{i-r}(\Gamma(m+i+2) / \Gamma(m-$ $i+1) \Gamma(2 i+2))(i ! /(i-r) ! r !), C_{r}^{j}=r ! / j !(r-j) !$.

Proof. The analytical form of the shifted Chebyshev polynomials [30] of the second-kind $U_{m}^{*}$ of degree $m$ is given by

$$
\begin{aligned}
U_{m}^{*}(x) & =\sum_{i=0}^{m}(-1)^{i} 2^{2 m-2 i} \frac{\Gamma(2 m-i+2)}{\Gamma(i+1) \Gamma(2 m-2 i+2)} x^{m-i} \\
& =\sum_{i=0}^{m}(-1)^{m-i} 2^{2 i} \frac{\Gamma(m+i+2)}{\Gamma(m-i+1) \Gamma(2 i+2)} x^{i} .
\end{aligned}
$$

According to the relation between $U_{m}^{*}(x)$ and $U_{m}(x)$, it gives that

$$
\begin{aligned}
& U_{m}\left(2^{k} x-2 n+1\right)=\sum_{i=0}^{m}(-1)^{m-i} 2^{2 i} \\
& \cdot \frac{\Gamma(m+i+2)}{\Gamma(m-i+1) \Gamma(2 i+2)}\left(2^{k-1} x-(n-1)\right)^{i} \\
& =\sum_{i=0}^{m}(-1)^{m-i} 2^{2 i} \frac{\Gamma(m+i+2)}{\Gamma(m-i+1) \Gamma(2 i+2)} 2^{i(k-1)}(x \\
& \left.-\frac{n-1}{2^{k-1}}\right)^{i}=\sum_{i=0}^{m}(-1)^{m-i} 2^{2 i} \\
& \cdot \frac{\Gamma(m+i+2)}{\Gamma(m-i+1) \Gamma(2 i+2)} 2^{i(k-1)} \sum_{r=0}^{i} C_{i}^{r} x^{i-r}\left(-\frac{n-1}{2^{k-1}}\right)^{r} \\
& =\sum_{i=0}^{m} \sum_{r=0}^{i}(-1)^{m-i+r} 2^{2 i} 2^{(i-r)(k-1)}(n-1)^{r} \\
& \cdot \frac{\Gamma(m+i+2)}{\Gamma(m-i+1) \Gamma(2 i+2)} \frac{i !}{r !(i-r) !} x^{i-r} .
\end{aligned}
$$

By interchanging the summation and substituting $i-r$ with $r, U_{m}\left(2^{k} x-2 n+1\right)$ can be written as

$$
\begin{aligned}
& U_{m}\left(2^{k} x-2 n+1\right)=\sum_{r=0}^{m} \sum_{i=r}^{m}(-1)^{m-r} \\
& \cdot 2^{2 i} 2^{r(k-1)}(n-1)^{i-r} \\
& \cdot \frac{\Gamma(m+i+2)}{\Gamma(m-i+1) \Gamma(2 i+2)} \frac{i !}{(i-r) ! r !} x^{r} .
\end{aligned}
$$

$$
I^{\alpha} \psi_{n, m}(x)= \begin{cases}0, & x<\frac{n-1}{2^{k-1}}, \\ \frac{1}{\Gamma(\alpha)} \int_{(n-1) / 2^{k-1}}^{x}(x-t)^{\alpha-1} \psi_{n, m}(t) d t, & \frac{n-1}{2^{k-1}} \leq x \leq \frac{n}{2^{k-1}}, \\ \frac{1}{\Gamma(\alpha)} \int_{(n-1) / 2^{k-1}}^{n / 2^{k-1}}(x-t)^{\alpha-1} \psi_{n, m}(t) d t, & x>\frac{n}{2^{k-1}},\end{cases}
$$

Let $T_{i, i-r}^{m, n, k}=(-1)^{m-r} 2^{2 i} 2^{r(k-1)}(n-1)^{i-r}(\Gamma(m+i+2) / \Gamma(m-$ $i+1) \Gamma(2 i+2))(i ! /(i-r) ! r !)$; then

$$
U_{m}\left(2^{k} x-2 n+1\right)=\sum_{r=0}^{m} \sum_{i=r}^{m} T_{i, i-r}^{m, n, k} x^{r} .
$$

Therefore

$$
\begin{aligned}
& \psi_{n, m}(x) \\
& = \begin{cases}2^{k / 2} \sqrt{\frac{2}{\pi}} \sum_{r=0}^{m} \sum_{i=r}^{m} T_{i, i-r}^{m, n, k} x^{r}, & \frac{n-1}{2^{k-1}} \leq x<\frac{n}{2^{k-1}} . \\
0, & \text { otherwise. }\end{cases}
\end{aligned}
$$

So, when $(n-1) / 2^{k-1} \leq x \leq n / 2^{k-1}$, let $u=x-t$; then

$$
\begin{aligned}
I^{\alpha} x^{r} & =\frac{1}{\Gamma(\alpha)} \int_{(n-1) / 2^{k-1}}^{x}(x-t)^{\alpha-1} t^{r} d t \\
& =\frac{1}{\Gamma(\alpha)} \int_{0}^{x-(n-1) / 2^{k-1}} u^{\alpha-1}(x-u)^{r} d u \\
& =\frac{1}{\Gamma(\alpha)} \int_{0}^{x-(n-1) / 2^{k-1}} u^{\alpha-1} \sum_{j=0}^{r} C_{r}^{j} x^{r-j}(-u)^{j} d u \\
& =\frac{1}{\Gamma(\alpha)} \sum_{j=0}^{r} \frac{(-1)^{j}}{j+\alpha} C_{r}^{j} x^{r-j}\left(x-\frac{n-1}{2^{k-1}}\right)^{j+\alpha} .
\end{aligned}
$$

Similarly, when $x>n / 2^{k-1}$, we have

$$
\begin{aligned}
& I^{\alpha} x^{r}=\frac{1}{\Gamma(\alpha)} \int_{(n-1) / 2^{k-1}}^{n / 2^{k-1}}(x-t)^{\alpha-1} t^{r} d t=\frac{1}{\Gamma(\alpha)} \\
& \cdot \int_{x-n / 2^{k-1}}^{x-(n-1) / 2^{k-1}} u^{\alpha-1}(x-u)^{r} d u=\frac{1}{\Gamma(\alpha)} \\
& \cdot \int_{x-n / 2^{k-1}}^{x-(n-1) / 2^{k-1}} u^{\alpha-1} \sum_{j=0}^{r} C_{r}^{j} x^{r-j}(-u)^{j} d u=\frac{1}{\Gamma(\alpha)} \\
& \cdot \sum_{j=0}^{r} \frac{(-1)^{j}}{j+\alpha} C_{r}^{j} x^{r-j}\left[\left(x-\frac{n-1}{2^{k-1}}\right)^{j+\alpha}\right. \\
& \left.\quad-\left(x-\frac{n}{2^{k-1}}\right)^{j+\alpha}\right] .
\end{aligned}
$$

Applying the Riemann-Liouville fractional integral of order $\alpha$ with respect to $x$ on $\psi_{n, m}(x)$, we obtain 


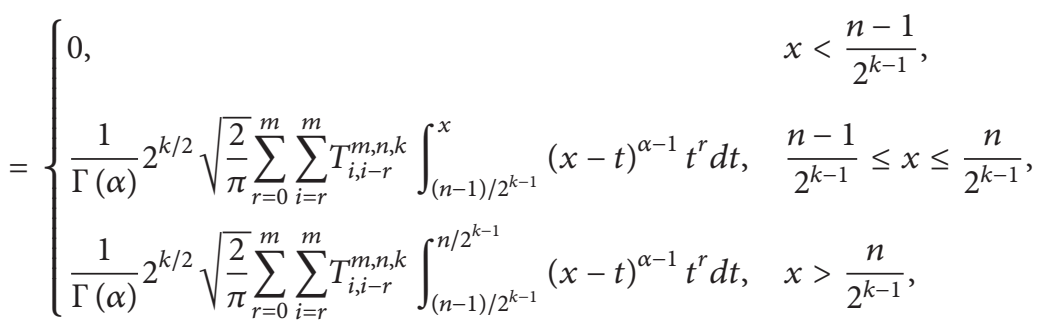

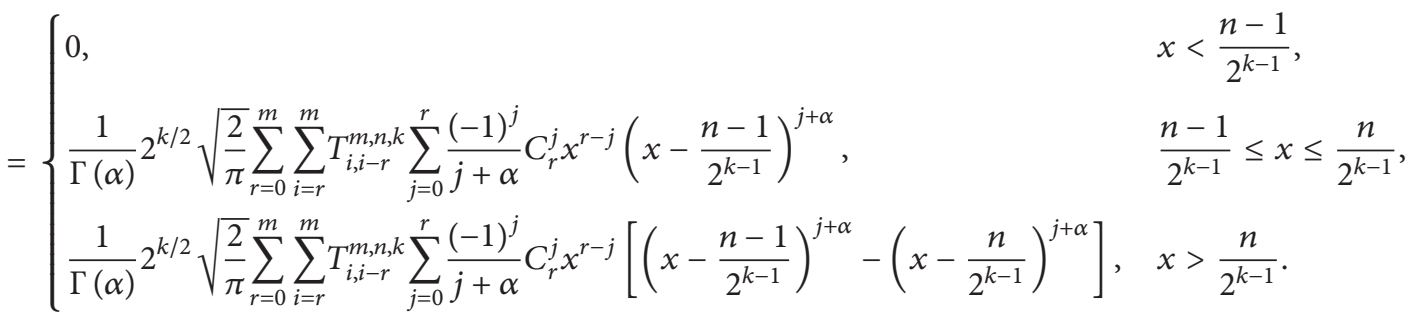

Thus, we have

$$
I^{\alpha} \psi_{n, m}(x)= \begin{cases}0, & x<\frac{n-1}{2^{k-1}}, \\ \frac{1}{\Gamma(\alpha)} 2^{k / 2} \sqrt{\frac{2}{\pi}} \sum_{r=0}^{m} \sum_{i=r}^{m} \sum_{j=0}^{r} T_{i, i-r}^{m, n, k} \frac{(-1)^{j}}{j+\alpha} C_{r}^{j}\left(x-\frac{n-1}{2^{k-1}}\right)^{j+\alpha}, & \frac{n-1}{2^{k-1}} \leq x \leq \frac{n}{2^{k-1}} \\ \frac{1}{\Gamma(\alpha)} 2^{k / 2} \sqrt{\frac{2}{\pi}} \sum_{r=0}^{m} \sum_{i=r}^{m} \sum_{j=0}^{r} T_{i, i-r}^{m, n, k} \frac{(-1)^{j}}{j+\alpha} C_{r}^{j} x^{r-j}\left[\left(x-\frac{n-1}{2^{k-1}}\right)^{j+\alpha}-\left(x-\frac{n}{2^{k-1}}\right)^{j+\alpha}\right], & x>\frac{n}{2^{k-1}} .\end{cases}
$$

The proof is completed.

For example, in the case of $k=2, M=3, x=0.65, \alpha=$ 1.7 , we obtain

$$
I^{\alpha} \Psi_{6 \times 1}(0.65)=\left(\begin{array}{c}
0.455620526461909 \\
-0.13717577587602 \\
0.144459099033891 \\
0.041066248466599 \\
-0.0638808309480429 \\
0.0620309999360338
\end{array}\right),
$$

where

$$
\begin{aligned}
& \Psi_{6 \times 1}(x) \\
& =\left(\psi_{1,0}(x) \quad \psi_{1,1}(x) \quad \psi_{1,2}(x) \quad \psi_{2,0}(x) \quad \psi_{2,1}(x) \quad \psi_{2,2}(x)\right)^{T} .
\end{aligned}
$$

\section{Description of the Proposed Method}

Consider the time-fractional diffusion-wave equation with the following form:

$$
\begin{aligned}
\frac{\partial^{\alpha} \mu(x, t)}{\partial t^{\alpha}}+\frac{\partial \mu(x, t)}{\partial t} & =\frac{\partial \mu^{2}(x, t)}{\partial x^{2}}+q(x, t), \\
0 & \leq x \leq 1,0 \leq t \leq 1,1<\alpha \leq 2,
\end{aligned}
$$

with initial condition

$$
\begin{aligned}
& \mu(x, 0)=f_{0}(x), \\
& \mu_{t}(x, 0)=f_{1}(x) \\
& 0 \leq x \leq 1,
\end{aligned}
$$

and boundary conditions

$$
\begin{aligned}
& \mu(0, t)=g_{0}(t), \\
& \mu(1, t)=g_{1}(t),
\end{aligned}
$$

$$
0 \leq t \leq 1
$$

where $f_{0}(\cdot), f_{1}(\cdot), g_{0}(\cdot), g_{1}(\cdot)$ are given functions with secondorder continuous derivatives in $L^{2}[0,1)$ and $q(\cdot, \cdot)$ is a given function in $L^{2}([0,1) \times[0,1))$.

To solve this problem, we assume

$$
\frac{\partial^{4} \mu(x, t)}{\partial x^{2} \partial t^{2}} \approx \Psi^{T}(x) \cdot U \cdot \Psi(t),
$$

where $U=\left(u_{i, j}\right)_{2^{k-1} M \times 2^{k-1} M}$ is an unknown matrix which should be determined and $\Psi(\cdot)$ is as in (13). By integrating 
two times with respect to $t$ on both sides of (52) and together with (2), we have

$$
\begin{aligned}
\frac{\partial^{2} \mu(x, t)}{\partial x^{2}} \approx & f_{0}^{\prime \prime}(x)+t f_{1}^{\prime \prime}(x)+\Psi^{T}(x) \cdot U \\
& \cdot\left(I^{2} \Psi(t)\right)
\end{aligned}
$$

Also by integrating (53) two times with respect to $x$, we obtain

$$
\begin{aligned}
\frac{\partial \mu(x, t)}{\partial x}= & \left.\frac{\partial \mu(x, t)}{\partial x}\right|_{x=0}+f_{0}^{\prime}(x)-f_{0}^{\prime}(0) \\
& +t\left(f_{1}^{\prime}(x)-f_{1}^{\prime}(0)\right)+(I \Psi(x))^{T} \cdot U \\
& \cdot\left(I^{2} \Psi(t)\right), \\
\mu(x, t) \approx & \mu(0, t)+\left.x \frac{\partial \mu(x, t)}{\partial x}\right|_{x=0} \\
& +\left(f_{0}(x)-f_{0}(0)-x f_{0}^{\prime}(0)\right) \\
& +t\left(f_{1}(x)-f_{1}(0)-x f_{1}^{\prime}(0)\right) \\
& +\left(I^{2} \Psi(x)\right)^{T} \cdot U \cdot\left(I^{2} \Psi(t)\right) .
\end{aligned}
$$

Putting $x=1$ in (55) and considering the boundary conditions (51), we get

$$
\begin{aligned}
\mu(1, t) \approx & \mu(0, t)+\left.\frac{\partial \mu(x, t)}{\partial x}\right|_{x=0} \\
& +\left(f_{0}(1)-f_{0}(0)-f_{0}^{\prime}(0)\right) \\
& +t\left(f_{1}(1)-f_{1}(0)-f_{1}^{\prime}(0)\right)+\left(I^{2} \Psi(1)\right)^{T} \\
& \cdot U \cdot\left(I^{2} \Psi(t)\right) .
\end{aligned}
$$

Thus, we have

$$
\begin{aligned}
\left.\frac{\partial \mu(x, t)}{\partial x}\right|_{x=0} \approx & g_{1}(t)-g_{0}(t) \\
& -\left(f_{0}(1)-f_{0}(0)-f_{0}^{\prime}(0)\right) \\
& -t\left(f_{1}(1)-f_{1}(0)-f_{1}^{\prime}(0)\right) \\
& -\left(I^{2} \Psi(1)\right)^{T} \cdot U \cdot\left(I^{2} \Psi(t)\right) .
\end{aligned}
$$

Write

$$
\begin{aligned}
H(t)= & g_{1}(t)-g_{0}(t)-\left(f_{0}(1)-f_{0}(0)-f_{0}^{\prime}(0)\right) \\
& -t\left(f_{1}(1)-f_{1}(0)-f_{1}^{\prime}(0)\right)-\left(I^{2} \Psi(1)\right)^{T} \\
& \cdot U \cdot\left(I^{2} \Psi(t)\right) .
\end{aligned}
$$

So

$$
\begin{aligned}
\mu(x, t) \approx & g_{0}(t)+x H(t)+\left(f_{0}(x)-f_{0}(0)-x f_{0}^{\prime}(0)\right) \\
& +t\left(f_{1}(x)-f_{1}(0)-x f_{1}^{\prime}(0)\right) \\
& +\left(I^{2} \Psi(x)\right)^{T} \cdot U \cdot\left(I^{2} \Psi(t)\right) .
\end{aligned}
$$

Applying fractional differentiation of order $\alpha$ and order one on both sides of (59) with respect to $t$, we get

$$
\begin{aligned}
\frac{\partial \mu^{\alpha}(x, t)}{\partial t^{\alpha}} \approx & D^{\alpha} g_{0}(t)+x D^{\alpha} H(t)+\left(I^{2} \Psi(x)\right)^{T} \cdot U \\
& \cdot\left(I^{2-\alpha} \Psi(t)\right), \\
\frac{\partial \mu(x, t)}{\partial t} \approx & g_{0}^{\prime}(t)+x H^{\prime}(t) \\
& +\left(f_{1}(x)-f_{1}(0)-x f_{1}^{\prime}(0)\right) \\
& +\left(I^{2} \Psi(x)\right)^{T} \cdot U \cdot(I \Psi(t)),
\end{aligned}
$$

where

$$
\begin{aligned}
D^{\alpha} H(t)= & D^{\alpha} g_{1}(t)-D^{\alpha} g_{0}(t)-\left(I^{2} \Psi(1)\right)^{T} \cdot U \\
& \cdot\left(I^{2-\alpha} \Psi(t)\right), \\
H^{\prime}(t)= & g_{1}^{\prime}(t)-g_{0}^{\prime}(t)-\left(f_{1}(1)-f_{1}(0)-f_{1}^{\prime}(0)\right) \\
& -\left(I^{2} \Psi(1)\right)^{T} \cdot U \cdot(I \Psi(t)) .
\end{aligned}
$$

Now by substituting (53), (59), and (60) into (49), replacing $\approx$ by $=$, and taking collocation points $x_{i}=(2 i-1) / 2^{k} M, t_{j}=$ $(2 j-1) / 2^{k} M, i, j=1,2, \ldots, 2^{k-1} M$, we obtain the following linear system of algebraic equations:

$$
\begin{aligned}
& D^{\alpha} g_{0}\left(t_{j}\right)+x_{i} D^{\alpha} H\left(t_{j}\right)+\left(I^{2} \Psi\left(x_{i}\right)\right)^{T} \cdot U \\
& \cdot\left(I^{2-\alpha} \Psi\left(t_{j}\right)\right)+\left(g_{0}^{\prime}\left(t_{j}\right)+x_{i} H^{\prime}\left(t_{j}\right)\right. \\
& \quad+\left(f_{1}\left(x_{i}\right)-f_{1}(0)-x_{i} f_{1}^{\prime}(0)\right)+\left(I^{2} \Psi\left(x_{i}\right)\right)^{T} \cdot U \\
&\left.\quad \cdot\left(\Psi\left(t_{j}\right)\right)\right)-\left(f_{0}^{\prime \prime}\left(x_{i}\right)+t_{j} f_{1}^{\prime \prime}\left(x_{i}\right)+\Psi^{T}\left(x_{i}\right) \cdot U\right. \\
&\left.\quad \cdot I^{2} \Psi\left(t_{j}\right)\right)=q\left(x_{i}, t_{j}\right),
\end{aligned}
$$

$i, j=1,2, \ldots, 2^{k-1} M$. By solving this system to determine the unknown matrix $U$, we can achieve an approximate solution for the problem by substituting $U$ into (59).

\section{Numerical Examples}

In this section, we give some numerical examples to demonstrate the efficiency and reliability of the proposed method. In all the examples, the package of Matlab 2016a has been used.

Example 1. Consider the following time-fractional diffusionwave equation ([17]) of order $\alpha(1<\alpha \leq 2)$

$$
\begin{aligned}
& \frac{\partial^{\alpha} \mu(x, t)}{\partial t^{\alpha}}+\frac{\partial \mu(x, t)}{\partial t}=\frac{\partial^{2} \mu(x, t)}{\partial x^{2}}+q(x, t), \\
& 0<x<1,0<t \leq 1,
\end{aligned}
$$




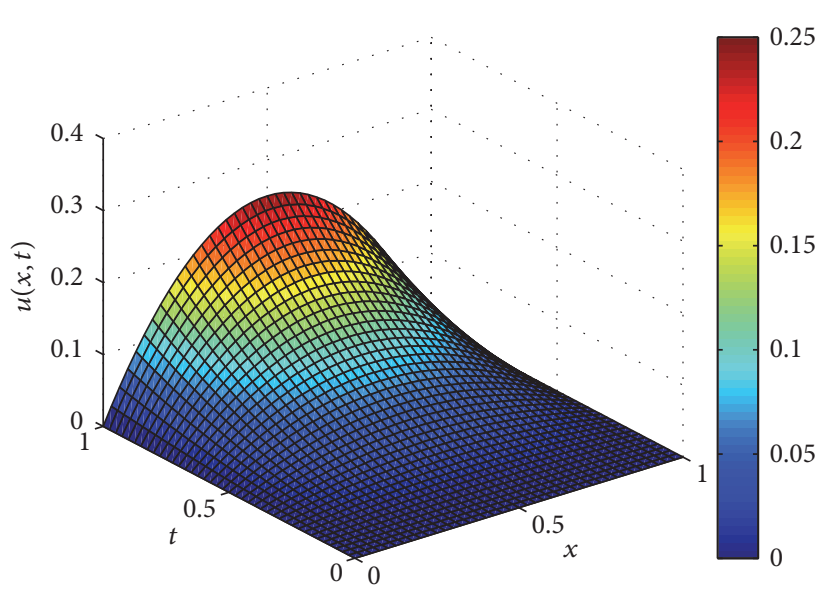

(a)

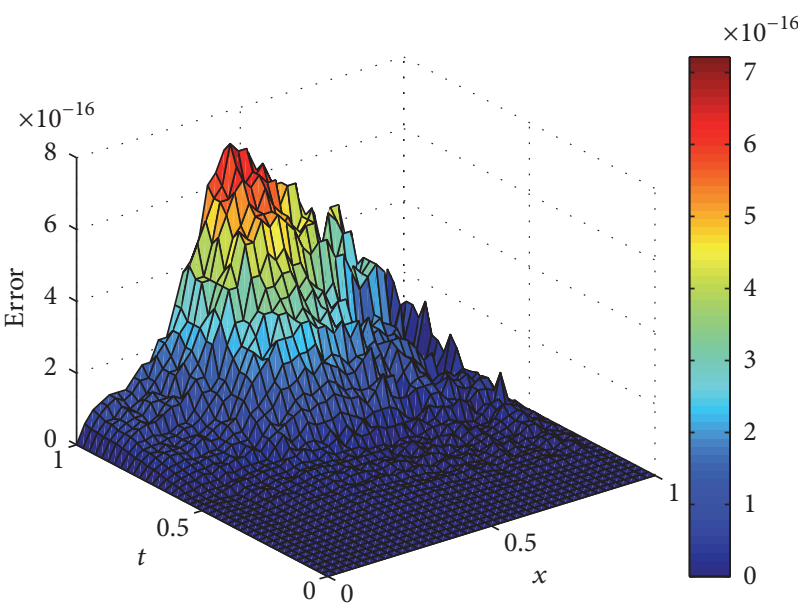

(b)

FIgURE 1: Approximate solution (a) and absolute error (b) for Example 1 with $\alpha=1.1, k=2$, and $M=3$.

TABLE 1: The absolute error for Example 1 for some different values $1<\alpha \leq 2$ at some different points.

\begin{tabular}{lccccc}
\hline$(x, t)$ & $\alpha=1.1$ & $\alpha=1.3$ & $\alpha=1.5$ & $\alpha=1.7$ & $\alpha=1.9$ \\
\hline$(0.1,0.1)$ & $3.25261 e-19$ & $4.33681 e-19$ & $3.25261 e-19$ & $8.67362 e-19$ & $1.19262 e-18$ \\
$(0.2,0.2)$ & $5.20417 e-18$ & $3.46945 e-18$ & $6.07153 e-18$ & $2.60209 e-18$ & $7.80626 e-18$ \\
$(0.3,0.3)$ & $1.38778 e-17$ & $6.93889 e-18$ & $2.77556 e-17$ & $6.93889 e-18$ & $1.38778 e-17$ \\
$(0.4,0.4)$ & $2.77556 e-17$ & $1.38778 e-17$ & $5.55112 e-17$ & $3.46945 e-17$ & $2.77556 e-17$ \\
$(0.5,0.5)$ & $2.77556 e-17$ & 0 & $8.32667 e-17$ & $3.46945 e-17$ & $4.16334 e-17$ \\
$(0.6,0.6)$ & $6.93889 e-17$ & $4.16334 e-17$ & $2.35922 e-16$ & $1.38778 e-17$ & $6.93889 e-17$ \\
$(0.7,0.7)$ & $1.66533 e-16$ & $1.38778 e-16$ & $3.60822 e-16$ & $5.55112 e-17$ & $2.77556 e-17$ \\
$(0.8,0.8)$ & $1.38778 e-16$ & $1.94289 e-16$ & $4.16334 e-16$ & $2.77556 e-17$ & $1.38778 e-16$ \\
$(0.9,0.9)$ & $1.38778 e-17$ & $1.24900 e-16$ & $3.19189 e-16$ & $1.24900 e-16$ & $1.24900 e-16$ \\
\hline
\end{tabular}

TABle 2: The absolute error for Example 1 for some different values $1<\alpha \leq 2$ at some different points in [17].

\begin{tabular}{lccccc}
\hline$(x, t)$ & $\alpha=1.1$ & $\alpha=1.3$ & $\alpha=1.5$ & $\alpha=1.7$ & $\alpha=1.9$ \\
\hline$(0.1,0.1)$ & $4.3939 e-6$ & $1.3694 e-5$ & $2.2112 e-5$ & $2.3491 e-5$ & $1.2825 e-5$ \\
$(0.2,0.2)$ & $7.5711 e-6$ & $2.2302 e-5$ & $3.7623 e-5$ & $4.6694 e-5$ & $3.1949 e-5$ \\
$(0.3,0.3)$ & $8.3240 e-6$ & $2.6323 e-5$ & $4.9656 e-5$ & $6.9397 e-5$ & $5.3169 e-5$ \\
$(0.4,0.4)$ & $7.5553 e-6$ & $2.4508 e-5$ & $4.9748 e-5$ & $6.7937 e-5$ & $6.8327 e-5$ \\
$(0.5,0.5)$ & $5.9012 e-6$ & $1.8821 e-5$ & $3.9629 e-5$ & $5.1193 e-5$ & $5.7208 e-5$ \\
$(0.6,0.6)$ & $4.4231 e-6$ & $1.3440 e-5$ & $2.7980 e-5$ & $2.9601 e-5$ & $3.6814 e-5$ \\
$(0.7,0.7)$ & $2.9153 e-6$ & $8.1172 e-6$ & $1.5667 e-5$ & $8.8490 e-6$ & $1.7911 e-5$ \\
$(0.8,0.8)$ & $1.6695 e-6$ & $4.1365 e-6$ & $5.1427 e-6$ & $2.8695 e-4$ & $1.2030 e-5$ \\
$(0.9,0.9)$ & $6.9982 e-7$ & $3.1435 e-6$ & $2.0508 e-4$ & 5
\end{tabular}

with the initial conditions

$$
\begin{aligned}
\mu(x, 0) & =0, \\
\mu_{t}(x, 0) & =0,
\end{aligned}
$$

$$
0 \leq x \leq 1
$$

and the boundary conditions

$$
\begin{aligned}
& \mu(0, t)=0, \\
& \mu(1, t)=0,
\end{aligned}
$$

$$
0<t \leq 1
$$

where $q(x, t)=\left(2 t^{2-\alpha} / \Gamma(3-\alpha)+2 t\right)\left(x-x^{2}\right)+2 t^{2}$. The exact solution of this problem is $\mu(x, t)=t^{2} x(1-x)$.

The problem is solved by the proposed method for $k=$ $2, M=3$ and the $\mathrm{CPU}$ time is 3.818 seconds. Figure 1 shows the approximate solution and the absolute error of this problem for $\alpha=1.1$. Table 1 gives the absolute errors for different values of $\alpha$ at different points. To make a comparison, in Table 2, we list the absolute error obtained by the Legendre wavelets method in [17] based on fractional operational matrix of integral with $k=3, M=3$. From Figure 1 and Table 1, it can be seen that the presented method is very efficient and accurate in solving this problem. 


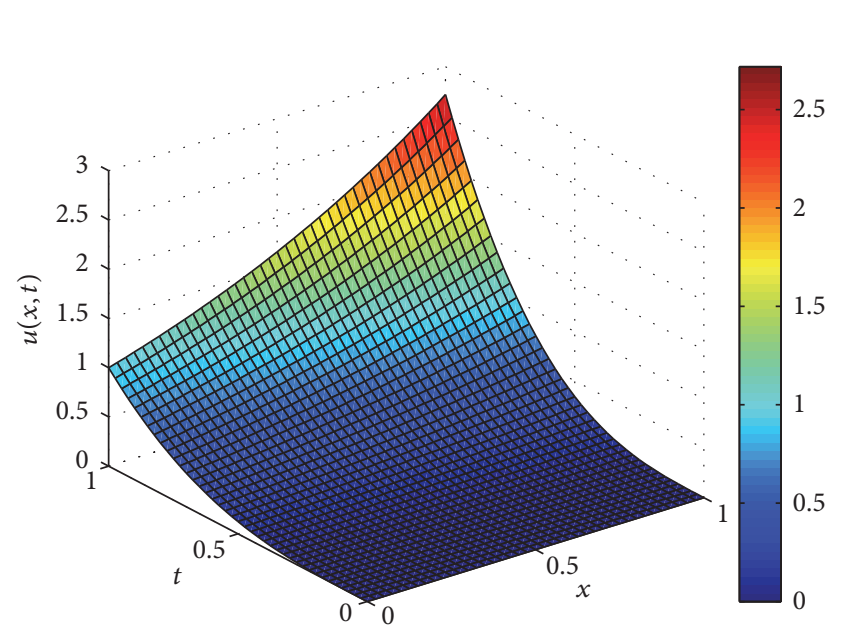

(a)

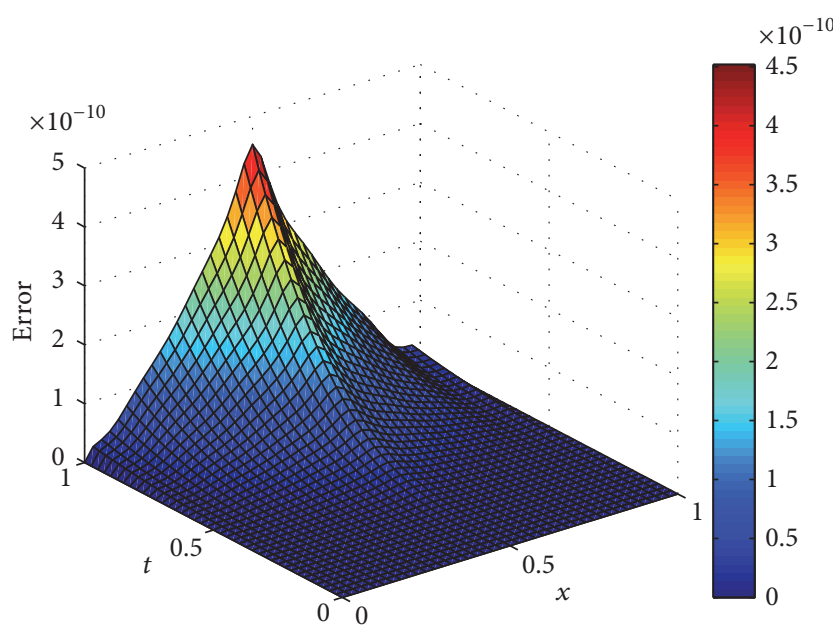

(b)

FIgURE 2: Approximate solution (a) and absolute error (b) for Example 2 with $\alpha=1.3, k=2$, and $M=6$.

TABLE 3: The absolute errors for Example 2 for some different values $1<\alpha \leq 2$ at some different points.

\begin{tabular}{|c|c|c|c|c|c|}
\hline$(x, t)$ & $\alpha=1.1$ & $\alpha=1.3$ & $\alpha=1.5$ & $\alpha=1.7$ & $\alpha=1.9$ \\
\hline$(0.1,0.1)$ & $3.94481 e-13$ & $3.86294 e-13$ & $3.59444 e-13$ & $3.55895 e-13$ & $3.40724 e-13$ \\
\hline$(0.2,0.2)$ & $2.10685 e-12$ & $1.30045 e-12$ & $8.35330 e-13$ & $7.96873 e-13$ & $9.32525 e-13$ \\
\hline$(0.3,0.3)$ & $1.80894 e-11$ & $1.19129 e-11$ & $6.13486 e-12$ & $1.57591 e-12$ & $1.36735 e-12$ \\
\hline$(0.4,0.4)$ & $1.32073 e-10$ & $1.12757 e-10$ & $9.26274 e-11$ & $7.36479 e-11$ & $5.68607 e-11$ \\
\hline$(0.5,0.5)$ & $3.59640 e-10$ & $3.20785 e-10$ & $2.78375 e-10$ & $2.35879 e-10$ & $1.96482 e-10$ \\
\hline$(0.6,0.6)$ & $2.96547 e-10$ & $2.39021 e-10$ & $1.74491 e-10$ & $1.07139 e-10$ & $4.29922 e-11$ \\
\hline$(0.7,0.7)$ & $5.52939 e-10$ & $4.83775 e-10$ & $4.04305 e-10$ & $3.17635 e-10$ & $2.30672 e-10$ \\
\hline$(0.8,0.8)$ & $5.01937 e-10$ & $4.34392 e-10$ & $3.55367 e-10$ & $2.65621 e-10$ & $1.69385 e-10$ \\
\hline$(0.9,0.9)$ & $9.61414 e-10$ & $9.16178 e-10$ & $8.62620 e-10$ & $7.99952 e-10$ & $7.29467 e-10$ \\
\hline
\end{tabular}

Example 2. Consider the following time-fractional diffusionwave equation ([17]) of order $\alpha(1<\alpha \leq 2)$

$$
\begin{aligned}
\frac{\partial^{\alpha} \mu(x, t)}{\partial t^{\alpha}}+\frac{\partial \mu(x, t)}{\partial t}=\frac{\partial^{2} \mu(x, t)}{\partial x^{2}} & +q(x, t), \\
0 & <x<1,0<t \leq 1,
\end{aligned}
$$

with the initial conditions

$$
\begin{gathered}
\mu(x, 0)=0, \\
\mu_{t}(x, 0)=0,
\end{gathered}
$$

$$
0 \leq x \leq 1
$$

and the boundary conditions

$$
\begin{aligned}
& \mu(0, t)=t^{3}, \\
& \mu(1, t)=e t^{3},
\end{aligned}
$$

where $q(x, t)=\left((6 / \Gamma(4-\alpha)) t^{3-\alpha}+3 t^{2}-t^{3}\right) e^{x}$. The exact solution of this problem is $\mu(x, t)=t^{3} e^{x}$.

The problem is solved by the proposed method for $k=$ 2, $M=6$ and the CPU time is 28.79 seconds. Figure 2 shows the approximate solution and the absolute error of this problem for $\alpha=1.3$. Table 3 gives the absolute error for different values of $\alpha$ at different points with $k=2, M=6$. In order to compare our results with obtained results in [17], we list the absolute error obtained by the Legendre wavelets method in [17] with $k=2, M=5$ in Table 4 . Table 5 lists the maximum absolute errors obtained by the proposed method for different choices of $M$ and $\alpha$ at the points $\left(x_{i}, t_{j}\right)$, where $x_{i}=i / 40, t_{j}=j / 40, i, j=0,1,2, \ldots, 40$. It is obvious that the results provided by the proposed method are more accurate than those given in [17].

Example 3. Consider the following time-fractional diffusionwave equation of order $\alpha(1<\alpha \leq 2)$ :

$$
\begin{aligned}
\frac{\partial^{\alpha} \mu(x, t)}{\partial t^{\alpha}}+\frac{\partial \mu(x, t)}{\partial t}=\frac{\partial^{2} \mu(x, t)}{\partial x^{2}} & +q(x, t), \\
0 & <x<1,0<t \leq 1,
\end{aligned}
$$


TABLE 4: The absolute errors for Example 2 for some different values $1<\alpha \leq 2$ at some different points in [17].

\begin{tabular}{|c|c|c|c|c|c|}
\hline$(x, t)$ & $\alpha=1.1$ & $\alpha=1.3$ & $\alpha=1.5$ & $\alpha=1.7$ & $\alpha=1.9$ \\
\hline$(0.1,0.1)$ & $6.7028 e-5$ & $6.2270 e-5$ & $6.0407 e-5$ & $4.9516 e-5$ & $2.0243 e-5$ \\
\hline$(0.2,0.2)$ & $1.8718 e-4$ & $1.6817 e-5$ & $1.5683 e-4$ & $1.3074 e-4$ & $5.9155 e-5$ \\
\hline$(0.3,0.3)$ & $3.0913 e-4$ & $2.8256 e-4$ & $2.7985 e-4$ & $2.3269 e-4$ & $1.0947 e-4$ \\
\hline$(0.4,0.4)$ & $4.0221 e-4$ & $3.6211 e-4$ & $3.7045 e-4$ & $3.4739 e-4$ & $1.6790 e-4$ \\
\hline$(0.5,0.5)$ & $4.5801 e-4$ & $3.8782 e-4$ & $3.8089 e-4$ & $3.2553 e-4$ & $1.6277 e-4$ \\
\hline$(0.6,0.6)$ & $4.5260 e-4$ & $3.7198 e-4$ & $3.6309 e-4$ & $3.1089 e-4$ & $1.9284 e-4$ \\
\hline$(0.7,0.7)$ & $4.0597 e-4$ & $3.0859 e-4$ & $3.2603 e-4$ & $4.6656 e-5$ & $6.2825 e-5$ \\
\hline$(0.8,0.8)$ & $3.1039 e-4$ & $7.9174 e-4$ & $6.5594 e-4$ & $1.2388 e-4$ & $1.0181 e-5$ \\
\hline$(0.9,0.9)$ & $1.7283 e-4$ & $2.1787 e-4$ & $7.1269 e-3$ & $1.6600 e-3$ & $2.0918 e-5$ \\
\hline
\end{tabular}

TABLE 5: Maximum absolute error for Example 2 with various choices of $M$ and $\alpha$.

\begin{tabular}{lccccc}
\hline$M$ & $\alpha=1.1$ & $\alpha=1.3$ & $\alpha=1.5$ & $\alpha=1.7$ & $\alpha=1.9$ \\
\hline 3 & $4.06786 e-6$ & $3.90708 e-6$ & $3.71627 e-6$ & $3.48835 e-6$ & $5.22168 e-6$ \\
4 & $6.03876 e-7$ & $5.76233 e-7$ & $5.43586 e-7$ & $3.50696 e-9$ & $4.59432 e-7$ \\
5 & $4.17452 e-9$ & $3.98846 e-9$ & $3.76854 e-9$ & $3.93658 e-10$ & $3.20222 e-9$ \\
6 & $4.74514 e-10$ & $4.51960 e-10$ & $4.25304 e-10$ & $1.98641 e-12$ & $3.56805 e-10$ \\
7 & $2.38765 e-12$ & $2.22533 e-12$ & $2.14806 e-12$ & $1.747305 e-13$ & $1.15907 e-13$ \\
8 & $2.42473 e-13$ & $2.90434 e-13$ & $3.26850 e-13$ & & 1.72 \\
\hline
\end{tabular}

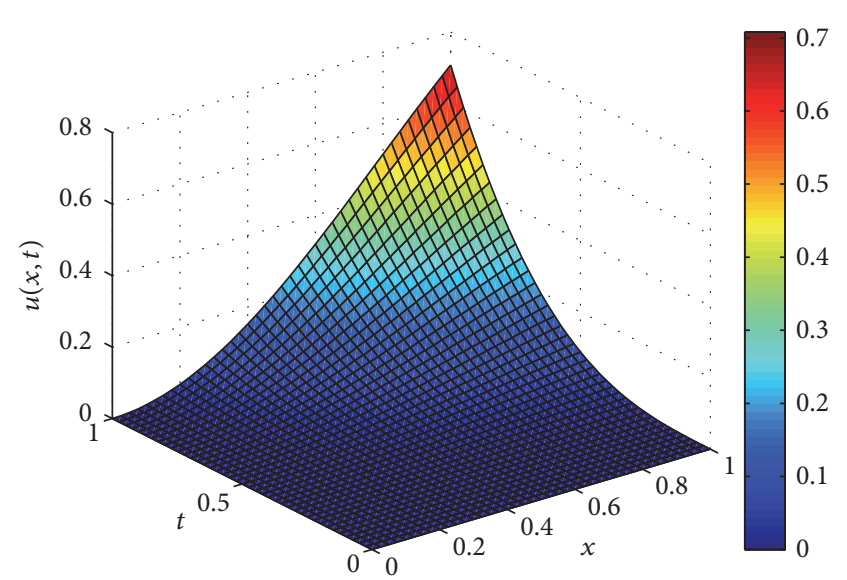

(a)

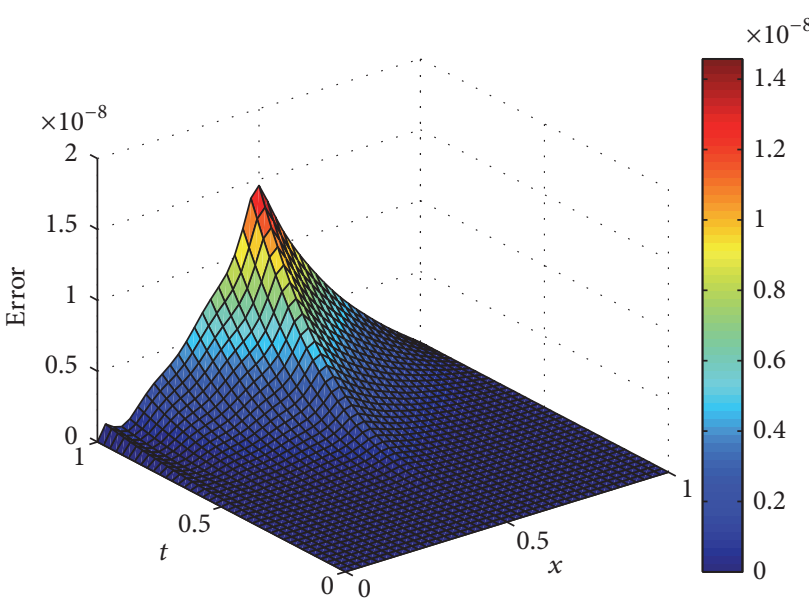

(b)

FIGURE 3: Approximate solution (a) and absolute error (b) for Example 3 with $\alpha=1.7, k=2$, and $M=6$.

with the initial conditions

$$
\begin{aligned}
\mu(x, 0) & =0, \\
\mu_{t}(x, 0) & =0,
\end{aligned}
$$

$$
0 \leq x \leq 1
$$

and the boundary conditions

$$
\begin{aligned}
& \mu(0, t)=0, \\
& \mu(1, t)=t^{3} \sin ^{2} 1,
\end{aligned}
$$

$$
0<t \leq 1
$$

where $q(x, t)=(\Gamma(4) / \Gamma(4-\alpha)) t^{3-\alpha} \sin ^{2} x+3 t^{2} \sin ^{2} x-$ $2 t^{3} \cos (2 x)$. The exact solution of this problem is $\mu(x, t)=$ $t^{3} \sin ^{2} x$

The problem is solved by the proposed method for $k=2$, $M=6$ and the CPU time is 28.42 seconds. Figure 3 shows the approximate solution and the absolute error of this problem in the case of $\alpha=1.7, k=2$, and $M=6$. Table 6 gives the absolute error of the approximate solutions for different values of $\alpha$ at different points with $k=2, M=6$. Table 7 lists the maximum absolute error obtained by the proposed method for different choices of $M$ and $\alpha$ at the points $\left(x_{i}, t_{j}\right)$, where $x_{i}=i / 40, t_{j}=j / 40, i, j=0,1,2, \ldots, 40$. 
TABLE 6: The absolute error for Example 3 for some different values $1<\alpha \leq 2$ at some different points.

\begin{tabular}{lccccc}
\hline$(x, t)$ & $\alpha=1.1$ & $\alpha=1.3$ & $\alpha=1.5$ & $\alpha=1.7$ & $\alpha=1.9$ \\
\hline$(0.1,0.1)$ & $5.49562 e-13$ & $5.47621 e-13$ & $4.98133 e-13$ & $4.68255 e-13$ & $5.26687 e-13$ \\
$(0.2,0.2)$ & $5.77200 e-12$ & $2.35711 e-12$ & $4.05830 e-13$ & $8.65065 e-14$ & $1.51648 e-14$ \\
$(0.3,0.3)$ & $8.08760 e-11$ & $5.48063 e-11$ & $3.10533 e-11$ & $1.33666 e-11$ & $3.69026 e-12$ \\
$(0.4,0.4)$ & $4.20186 e-10$ & $3.35630 e-10$ & $2.47962 e-10$ & $1.65984 e-10$ & $9.57750 e-11$ \\
$(0.5,0.5)$ & $1.64121 e-9$ & $1.46671 e-9$ & $1.27599 e-9$ & $1.08434 e-9$ & $9.04889 e-10$ \\
$(0.6,0.6)$ & $2.05951 e-9$ & $1.79826 e-9$ & $1.50447 e-9$ & $1.19672 e-9$ & $9.03980 e-10$ \\
$(0.7,0.7)$ & $2.38806 e-9$ & $2.07520 e-9$ & $1.71522 e-9$ & $1.32122 e-9$ & $9.24245 e-10$ \\
$(0.8,0.8)$ & $2.39668 e-9$ & $2.09691 e-9$ & $1.74583 e-9$ & $1.34653 e-9$ & $9.14931 e-10$ \\
$(0.9,0.9)$ & $1.75917 e-9$ & $1.56064 e-9$ & $1.32547 e-9$ & $1.04978 e-9$ & $7.37741 e-10$ \\
\hline
\end{tabular}

TABLe 7: Maximum absolute error for Example 3 with various choices of $M$ and $\alpha$.

\begin{tabular}{lccccc}
\hline$M$ & $\alpha=1.1$ & $\alpha=1.3$ & $\alpha=1.5$ & $\alpha=1.7$ & $\alpha=1.9$ \\
\hline 3 & $2.40504 e-5$ & $2.26983 e-5$ & $2.11041 e-5$ & $1.92403 e-5$ & $1.71638 e-5$ \\
4 & $3.99132 e-6$ & $3.77465 e-6$ & $3.51924 e-6$ & $3.22175 e-6$ & $2.89080 e-6$ \\
5 & $1.08639 e-7$ & $1.02446 e-7$ & $9.51434 e-8$ & $8.66286 e-8$ & $7.71522 e-8$ \\
6 & $1.23281 e-8$ & $1.16368 e-8$ & $1.08219 e-8$ & $9.87176 e-9$ & $8.81455 e-9$ \\
7 & $2.64246 e-10$ & $2.49148 e-10$ & $2.31377 e-10$ & $2.10629 e-10$ & $1.87484 e-10$ \\
8 & $3.21829 e-11$ & $3.06864 e-11$ & $2.88753 e-11$ & $2.66064 e-11$ & $2.40237 e-11$ \\
\hline
\end{tabular}

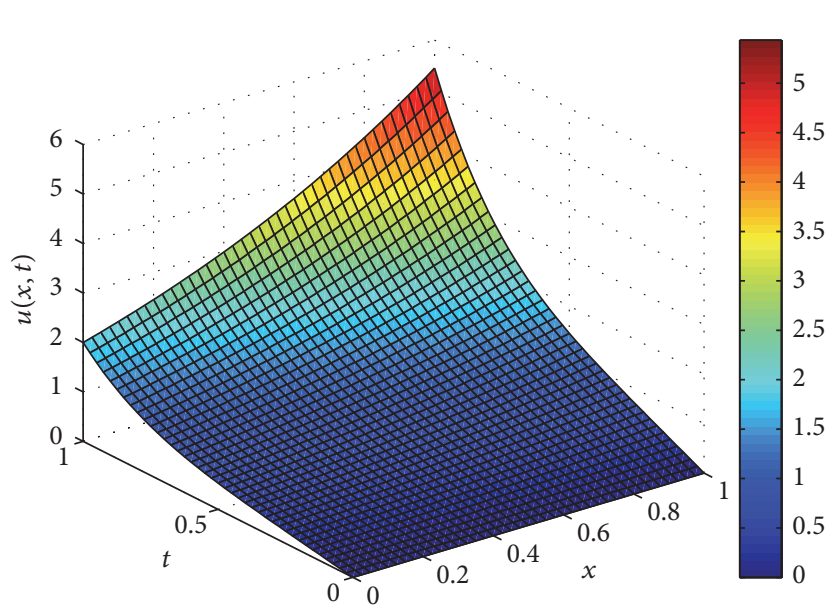

(a)

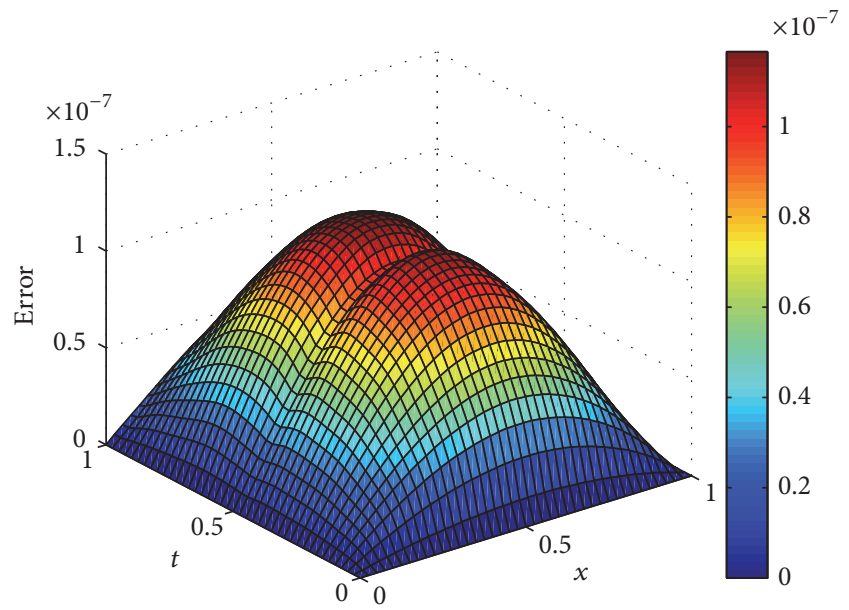

(b)

FIgURE 4: Approximate solution (a) and absolute error (b) for Example 4 with $\alpha=1.9, k=2$, and $M=6$.

Example 4. Consider the following time-fractional diffusionwave equation of order $\alpha(1<\alpha \leq 2)$

$$
\begin{aligned}
\frac{\partial^{\alpha} \mu(x, t)}{\partial t^{\alpha}}+\frac{\partial \mu(x, t)}{\partial t}=\frac{\partial^{2} \mu(x, t)}{\partial x^{2}} & +f(x, t), \\
0 & <x<1,0<t \leq 1,
\end{aligned}
$$

with the initial conditions

$$
\begin{aligned}
& \mu(x, 0)=0, \\
& \mu_{t}(x, 0)=e^{x}, \\
& 0 \leq x \leq 1,
\end{aligned}
$$

and the boundary conditions

$$
\begin{aligned}
& \mu(0, t)=t^{\alpha+3}+t, \\
& \mu(1, t)=\left(t^{\alpha+3}+t\right) e, \\
& \quad 0<t \leq 1,
\end{aligned}
$$

where $q(x, t)=\left((\Gamma(4+\alpha) / \Gamma(4)) t^{3}+(\alpha+3) t^{\alpha+2}+1-t^{\alpha+3}-t\right) e^{x}$. The exact solution of this problem is $\mu(x, t)=\left(t^{\alpha+3}+t\right) e^{x}$.

The space-time graph of the approximate solution and the absolute error for $\alpha=1.9, k=2$, and $M=6$ is presented in Figure 4. The absolute error for different values of $\alpha$ at different points with $k=2$ and $M=6$ 
TABLE 8: The absolute errors for Example 4 for some different values $1<\alpha \leq 2$ at some different points.

\begin{tabular}{lccccc}
\hline$(x, t)$ & $\alpha=1.1$ & $\alpha=1.3$ & $\alpha=1.5$ & $\alpha=1.7$ & $\alpha=1.9$ \\
\hline$(0.1,0.1)$ & $1.06076 e-9$ & $2.86893 e-9$ & $1.38103 e-8$ & $2.42944 e-8$ & $5.24253 e-8$ \\
$(0.2,0.2)$ & $1.40209 e-9$ & $6.00404 e-9$ & $2.27258 e-8$ & $7.25016 e-8$ & $4.98649 e-8$ \\
$(0.3,0.3)$ & $3.37278 e-9$ & $1.98379 e-9$ & $2.09526 e-8$ & $8.15413 e-8$ & $8.37632 e-8$ \\
$(0.4,0.4)$ & $2.36158 e-9$ & $9.26811 e-9$ & $2.79830 e-8$ & $6.65251 e-8$ & $1.06968 e-7$ \\
$(0.5,0.5)$ & $8.93198 e-8$ & $9.49760 e-8$ & $4.89761 e-8$ & $1.67284 e-7$ & $1.10555 e-7$ \\
$(0.6,0.6)$ & $1.44456 e-7$ & $2.91469 e-7$ & $2.53681 e-7$ & $1.66823 e-7$ & $9.46295 e-8$ \\
$(0.7,0.7)$ & $8.49482 e-8$ & $1.98030 e-7$ & $2.18826 e-7$ & $1.11109 e-7$ & $6.11390 e-8$ \\
$(0.8,0.8)$ & $3.27487 e-8$ & $9.88976 e-8$ & $1.30364 e-7$ & $4.77641 e-8$ & $2.47031 e-8$ \\
$(0.9,0.9)$ & $2.73139 e-9$ & $3.18928 e-8$ & $5.20473 e-8$ & &
\end{tabular}

TABLE 9: Maximum absolute error for Example 4 with various choices of $M$ and $\alpha$.

\begin{tabular}{lccccc}
\hline$M$ & $\alpha=1.1$ & $\alpha=1.3$ & $\alpha=1.5$ & $\alpha=1.7$ & $\alpha=1.9$ \\
\hline 3 & $2.27899 e-5$ & $6.43156 e-5$ & $9.16715 e-5$ & $1.32018 e-4$ & $1.02247 e-4$ \\
4 & $2.34479 e-6$ & $5.42695 e-6$ & $5.49280 e-6$ & $4.91082 e-6$ & $3.00590 e-6$ \\
5 & $4.92849 e-7$ & $1.03746 e-6$ & $9.65101 e-7$ & $7.05607 e-7$ & $3.62955 e-7$ \\
6 & $1.49081 e-7$ & $2.97338 e-7$ & $2.65557 e-7$ & $1.97476 e-7$ & $1.16463 e-7$ \\
7 & $5.83218 e-8$ & $1.08640 e-7$ & $8.96853 e-8$ & $5.56267 e-8$ & $4.37784 e-8$ \\
8 & $2.69626 e-8$ & $4.77286 e-8$ & $3.86373 e-8$ & $2.74826 e-8$ & $2.01824 e-8$ \\
\hline
\end{tabular}

TABLE 10: The absolute errors for Example 5 at some different points with $k=2$ and $M=3$ to 8 .

\begin{tabular}{lcccccc}
\hline$(x, t)$ & $M=3$ & $M=4$ & $M=5$ & $M=6$ & $M=7$ \\
\hline$(0.1,0.1)$ & $6.48090 e-8$ & $9.39323 e-9$ & $6.41101 e-10$ & $6.04636 e-11$ & $2.16316 e-12$ & $1.01696 e-13$ \\
$(0.2,0.2)$ & $2.27341 e-8$ & $5.69677 e-8$ & $7.56717 e-9$ & $1.05616 e-9$ & $9.40217 e-11$ & $1.22968 e-11$ \\
$(0.3,0.3)$ & $7.34805 e-6$ & $1.29090 e-6$ & $1.27698 e-7$ & $2.06609 e-8$ & $1.84093 e-9$ & $2.37917 e-10$ \\
$(0.4,0.4)$ & $3.45200 e-5$ & $6.82472 e-6$ & $7.01216 e-7$ & $1.09420 e-7$ & $9.84536 e-9$ & $1.27289 e-9$ \\
$(0.5,0.5)$ & $1.03114 e-4$ & $2.27987 e-5$ & $2.20253 e-6$ & $3.58064 e-7$ & $3.15850 e-8$ & $4.12741 e-9$ \\
$(0.6,0.6)$ & $8.61804 e-5$ & $1.96315 e-5$ & $2.03271 e-6$ & $3.53811 e-7$ & $3.28933 e-8$ & $4.39198 e-9$ \\
$(0.7,0.7)$ & $6.20577 e-5$ & $2.06432 e-5$ & $2.61296 e-6$ & $3.78036 e-7$ & $3.54969 e-8$ & $4.53937 e-9$ \\
$(0.8,0.8)$ & $2.11165 e-4$ & $1.58365 e-5$ & $2.34163 e-6$ & $3.37270 e-7$ & $3.52359 e-8$ & $4.14875 e-9$ \\
$(0.9,0.9)$ & $1.36820 e-4$ & $1.04491 e-6$ & $2.87015 e-6$ & $1.50481 e-7$ & $2.93362 e-8$ & $2.69187 e-9$ \\
\hline
\end{tabular}

is tabulated in Table 8. Table 9 lists the maximum absolute error obtained by the proposed method for different choices of $M$ and $\alpha$ at the points $\left(x_{i}, t_{j}\right)$, where $x_{i}=i / 40, t_{j}=$ $j / 40, i, j=0,1,2, \ldots, 40$. The CPU time for this problem is 28.32 seconds.

Example 5. Consider the following time-fractional diffusionwave equation:

$$
\begin{aligned}
& \frac{\partial^{1.5} \mu(x, t)}{\partial t^{1.5}}+\frac{\partial \mu(x, t)}{\partial t}=\frac{\partial^{2} \mu(x, t)}{\partial x^{2}}+q(x, t), \\
& 0<x<1,0<t \leq 1,
\end{aligned}
$$

with the initial conditions

$$
\begin{gathered}
\mu(x, 0)=e^{x^{2}}, \\
\mu_{t}(x, 0)=e^{x^{2}},
\end{gathered}
$$

$0 \leq x \leq 1$, and the boundary conditions

$$
\begin{aligned}
& \mu(0, t)=e^{t}, \\
& \mu(1, t)=e^{t+1}, \\
& 0<t \leq 1,
\end{aligned}
$$

where $q(x, t)=\left(\operatorname{erf}(\sqrt{t})-4 x^{2}-1\right) e^{x^{2}+t}$. The exact solution of this problem is $\mu(x, t)=e^{x^{2}+t}$.

This problem is solved by the proposed method with $k=$ $2, M=3$ to 8 . The absolute error of the approximate solutions for some different points are shown in Table 10. Figure 5 shows the approximate solution and the absolute error of this problem in the case of $k=2$ and $M=6$. The CPU time for this problem is 28.82 seconds.

From Figures 1-5 and Tables 1-10, it can be seen that the proposed method is very efficient and accurate in solving this problem and the obtained approximate solutions are very 


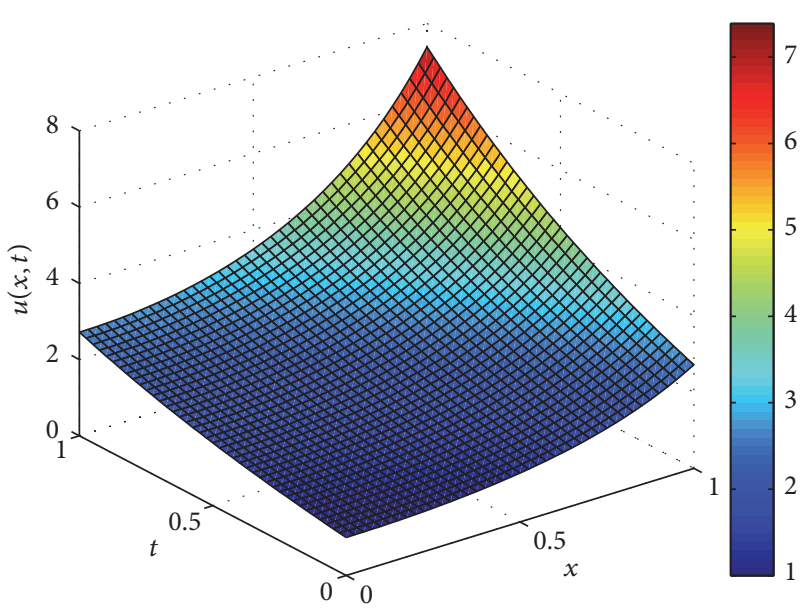

(a)

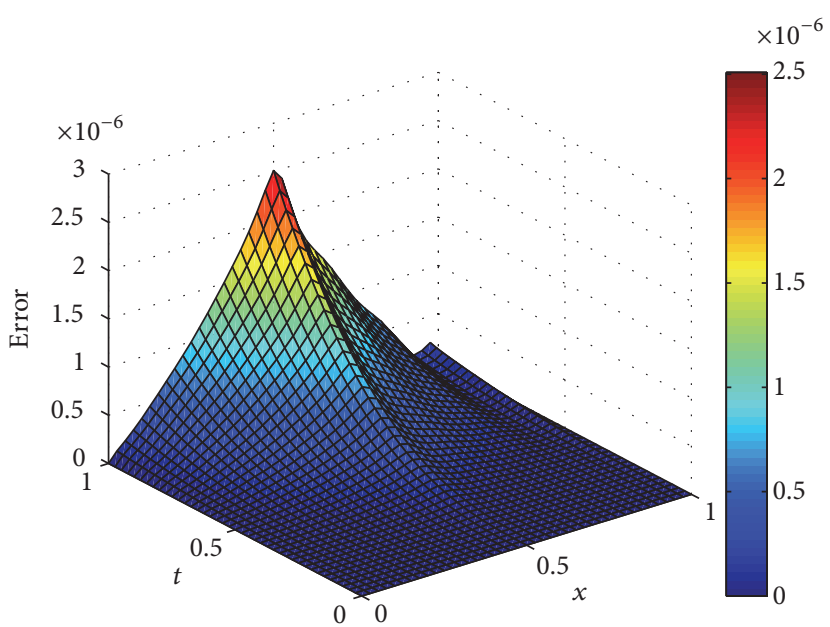

(b)

Figure 5: Approximate solution (a) and absolute error (b) for Example 5 with $k=2$ and $M=6$.

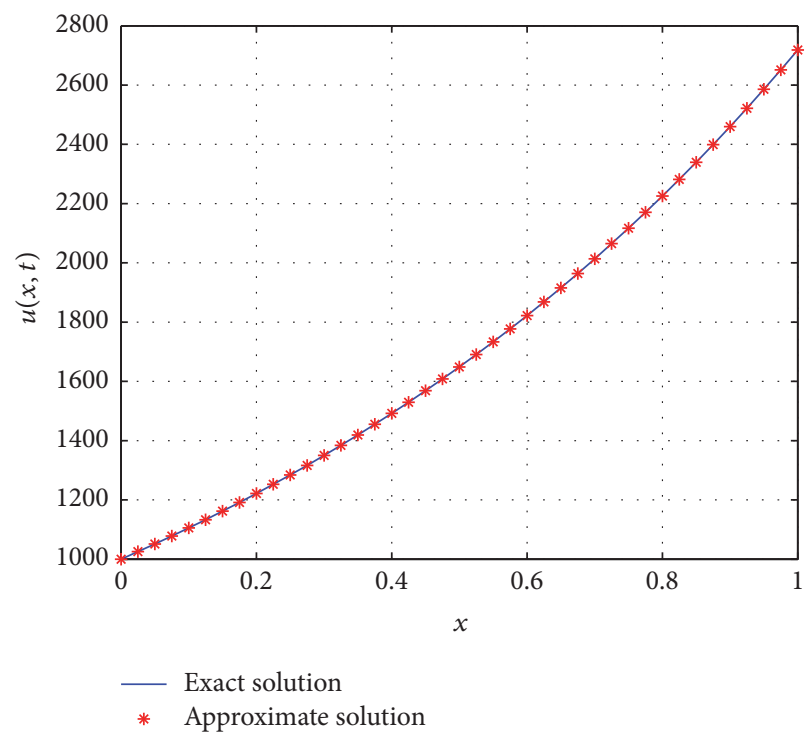

(a)

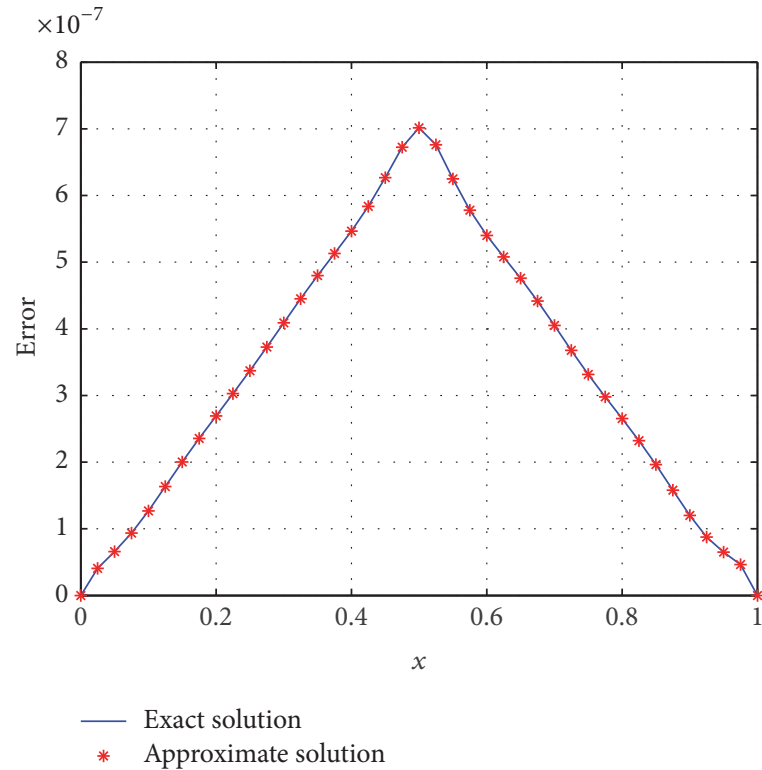

(b)

Figure 6: The exact and approximation solution (a) and corresponding error (b) at $k=2, M=6$, and $T=10$.

close to the exact ones for all chosen $\alpha$ with $1<\alpha \leq 2$. It is worth noting that more accurate result can be obtained by increasing basis functions.

Remark 6. It should be noted that the presented method can also be applied to the calculation of long time $T$. We only need to extend the second-kind Chebyshev wavelet basis functions on the interval $[0,1]$ to the general interval $[a, b]$. We choose Example 2 to illustrate the feasibility of this method. For example, Figure 6 shows the behavior of exact and approximation solution and corresponding error at $T=10$.

\section{Conclusion}

In this paper, the collocation method based on the secondkind Chebyshev wavelets has been applied to the timefractional diffusion-wave equations. We derived the fractional integral formula of a single Chebyshev wavelet in the Riemann-Liouville sense via the shifted Chebyshev polynomials of the second kind. Convergence and accuracy estimation of the second-kind Chebyshev wavelets expansion of two-dimension were given. The second-kind Chebyshev wavelets and their properties have been used to convert the problems under consideration into some corresponding 
linear systems of algebraic equations. The proposed method is very convenient for solving time-fractional diffusion-wave equations, since the boundary conditions are taken into account automatically during the process of establishing the expression of the approximate solution. Applicability and accuracy have been tested on some numerical examples. We also compared our results with the results obtained by Legendre wavelet, which showed that the proposed approach was more accurate. Furthermore, the proposed method can be expected to solve other types of fractional partial differential equations numerically.

\section{Conflicts of Interest}

The authors declare that they have no conflicts of interest.

\section{Authors' Contributions}

All authors contributed equally to the writing of this paper. All authors read and approved the manuscript.

\section{Acknowledgments}

This work is supported by the National Natural Science Foundation of China (Grant no. 11601076) and the Youth Science Foundation of Jiangxi Province (Grant nos. 20151BAB211004 and 20151BAB211012).

\section{References}

[1] J. Chen, F. Liu, V. Anh, S. Shen, Q. Liu, and C. Liao, “The analytical solution and numerical solution of the fractional diffusion-wave equation with damping," Applied Mathematics and Computation, vol. 219, no. 4, pp. 1737-1748, 2012.

[2] R. Darzi, B. Mohammadzade, S. Mousavi, and R. Beheshti, "Sumudu transform method for solving fractional differential equations and fractional diffusion-wave equation," Journal of Mathematics and Computer Science, vol. 2013, no. 6, pp. 79-84, 2013.

[3] S. S. Ray, "Exact solutions for time-fractional diffusion-wave equations by decomposition method," Physica Scripta, vol. 75, no. 1, pp. 53-61, 2007.

[4] J. Huang, Y. Tang, L. Vázquez, and J. Yang, "Two finite difference schemes for time fractional diffusion-wave equation," Numerical Algorithms, vol. 64, no. 4, pp. 707-720, 2013.

[5] F. Zeng, "Second-order stable finite difference schemes for the time-fractional diffusion-wave equation," Journal of Scientific Computing, vol. 65, no. 1, pp. 411-430, 2015.

[6] Z.-Z. Sun and X. Wu, "A fully discrete difference scheme for a diffusion-wave system," Applied Numerical Mathematics, vol. 56, no. 2, pp. 193-209, 2006.

[7] X. Hu and L. Zhang, "A compact finite difference scheme for the fourth-order fractional diffusion-wave system," Computer Physics Communications, vol. 182, no. 8, pp. 1645-1650, 2011.

[8] V. R. Hosseini, E. Shivanian, and W. Chen, "Local radial point interpolation (MLRPI) method for solving time fractional diffusion-wave equation with damping," Journal of Computational Physics, vol. 312, pp. 307-332, 2016.

[9] A. Esen, O. Tasbozan, Y. Ucar, and N. M. Yagmurlu, "A B-spline collocation method for solving fractional diffusion and fractional diffusion-wave equations," Tbilisi Mathematical Journal, vol. 8, no. 2, pp. 181-193, 2015.

[10] Z. Mao, A. Xiao, Z. Yu, and L. Shi, "Finite difference and sinccollocation approximations to a class of fractional diffusionwave equations," Journal of Applied Mathematics, vol. 2014, Article ID 536030, 11 pages, 2014.

[11] Z. Mao, A. Xiao, Z. Yu, and L. Shi, "Sinc-chebyshev collocation method for a class of fractional diffusion-wave equations," The Scientific World Journal, vol. 2014, Article ID 143983, 7 pages, 2014.

[12] S. Nemati and S. Sedaghat, "Matrix method based on the second kind Chebyshev polynomials for solving time fractional diffusion-wave equations," Journal of Applied Mathematics and Computing, vol. 51, no. 1-2, pp. 189-207, 2016.

[13] E. H. Doha, A. H. Bhrawy, and S. S. Ezz-Eldien, "A new Jacobi operational matrix: an application for solving fractional differential equations," Applied Mathematical Modelling, vol. 36, no. 10, pp. 4931-4943, 2012.

[14] A. H. Bhrawy, E. H. Doha, D. Baleanu, and S. S. Ezz-Eldien, "A spectral tau algorithm based on Jacobi operational matrix for numerical solution of time fractional diffusion-wave equations," Journal of Computational Physics, vol. 293, pp. 142-156, 2015.

[15] M. R. Hooshmandasl, M. H. Heydari, and C. Cattani, "Numerical solution of fractional sub-diffusion and time-fractional diffusion-wave equations via fractional-order Legendre functions," European Physical Journal Plus, vol. 131, no. 8, article 268, 2016.

[16] Z. Barikbin, "Two-dimensional Bernoulli wavelets with satisfier function in the Ritz-Galerkin method for the time fractional diffusion-wave equation with damping," Mathematical Sciences, vol. 11, no. 3, pp. 195-202, 2017.

[17] M. H. Heydari, M. R. Hooshmandasl, F. M. M. Maalek Ghaini, and C. Cattani, "Wavelets method for the time fractional diffusion-wave equation," Physics Letters A, vol. 379, no. 3, pp. 71-76, 2015.

[18] Y. Li, N. Sun, B. Zheng, Q. Wang, and Y. Zhang, "Wavelet operational matrix method for solving the Riccati differential equation," Communications in Nonlinear Science and Numerical Simulation, vol. 19, no. 3, pp. 483-493, 2014.

[19] S. Balaji, "Legendre wavelet operational matrix method for solution of fractional order Riccati differential equation," Journal of the Egyptian Mathematical Society, vol. 23, no. 2, pp. 263-270, 2015.

[20] M. H. Heydari, M. R. Hooshmandasl, and F. Mohammadi, "Legendre wavelets method for solving fractional partial differential equations with Dirichlet boundary conditions," Applied Mathematics and Computation, vol. 234, pp. 267-276, 2014.

[21] Y. Li, "Solving a nonlinear fractional differential equation using Chebyshev wavelets," Communications in Nonlinear Science and Numerical Simulation, vol. 15, no. 9, pp. 2284-2292, 2010.

[22] Y. Wang and L. Zhu, "Solving nonlinear Volterra integrodifferential equations of fractional order by using Euler wavelet method," Advances in Difference Equations, vol. 2017, article 27, 2017.

[23] L. Zhu and Q. Fan, "Solving fractional nonlinear Fredholm integro-differential equations by the second kind Chebyshev wavelet," Communications in Nonlinear Science and Numerical Simulation, vol. 17, no. 6, pp. 2333-2341, 2012.

[24] W. M. Abd-Elhameed, E. H. Doha, and Y. H. Youssri, "New wavelets collocation method for solving second-order multipoint boundary value problems using chebyshev polynomials 
of third and fourth kinds," Abstract and Applied Analysis, vol. 2013, Article ID 542839, 9 pages, 2013.

[25] Y. Chen, L. Sun, X. Li, and X. Fu, "Numerical solution of nonlinear fractional integral differential equations by using the second kind Chebyshev wavelets," Computer Modeling in Engineering and Sciences, vol. 90, no. 5, pp. 359-378, 2013.

[26] A. K. Gupta and S. Saha Ray, "Numerical treatment for the solution of fractional fifth-order Sawada-Kotera equation using second kind Chebyshev wavelet method," Applied Mathematical Modelling, vol. 39, no. 17, pp. 5121-5130, 2015.

[27] İ. Çelik, "Chebyshev wavelet collocation method for solving generalized Burgers-Huxley equation," Mathematical Methods in the Applied Sciences, vol. 39, no. 3, pp. 366-377, 2016.

[28] I. Podlubny, Fractional Differential Equations: An Introduction to Fractional Derivatives, Fractional Differential Equations, to Methods of Their Solution and Some of Their Applications, vol. 198 of Mathematics in Science and Engineering, Academic Press, New York, NY, USA, 1999.

[29] F. Zhou and X. Xu, "Numerical solution of the convection diffusion equations by the second kind Chebyshev wavelets," Applied Mathematics and Computation, vol. 247, pp. 353-367, 2014.

[30] N. H. Sweilam, A. M. Nagy, and A. A. El-Sayed, "Second kind shifted Chebyshev polynomials for solving space fractional order diffusion equation," Chaos, Solitons and Fractals, vol. 73, pp. 141-147, 2015. 


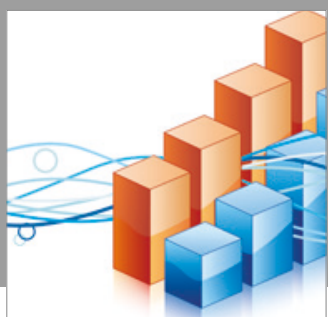

Advances in

Operations Research

vatersals

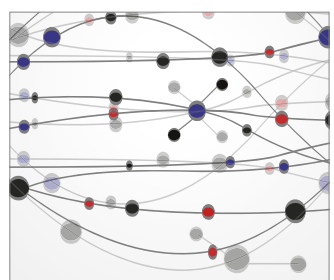

\section{The Scientific} World Journal
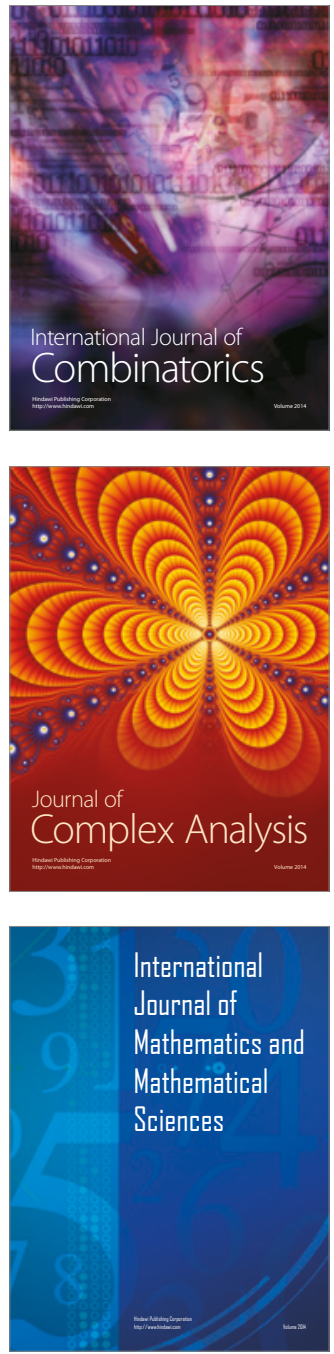
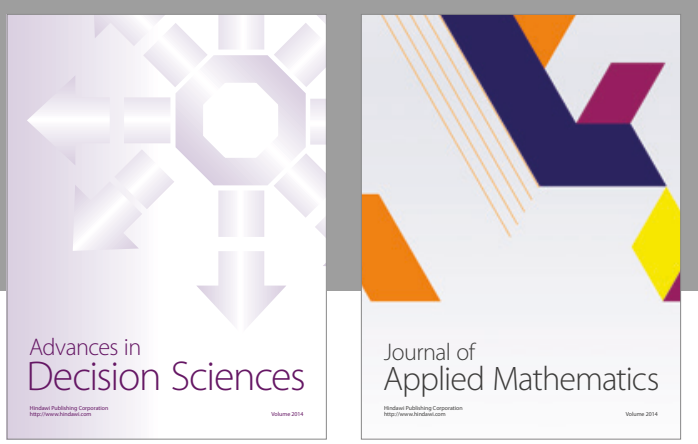

Algebra

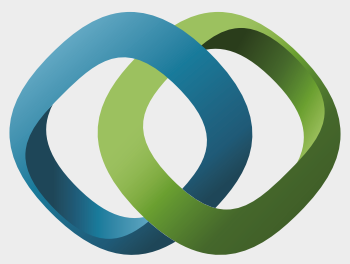

\section{Hindawi}

Submit your manuscripts at

https://www.hindawi.com
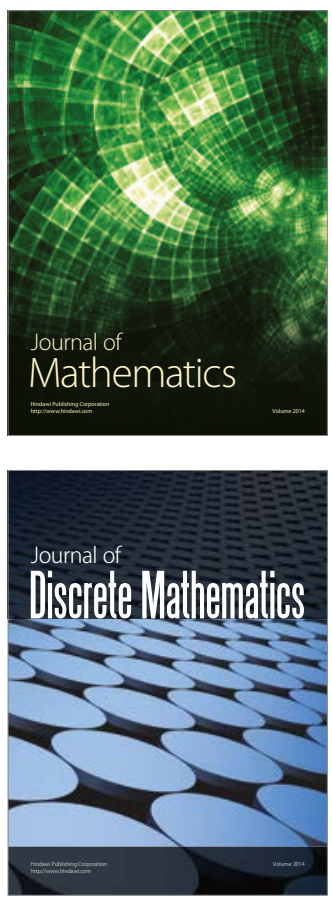

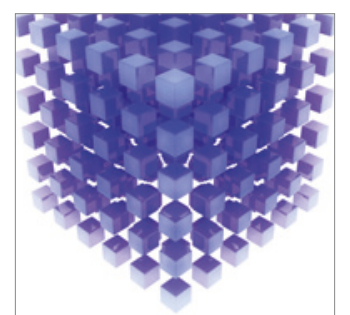

Mathematical Problems in Engineering
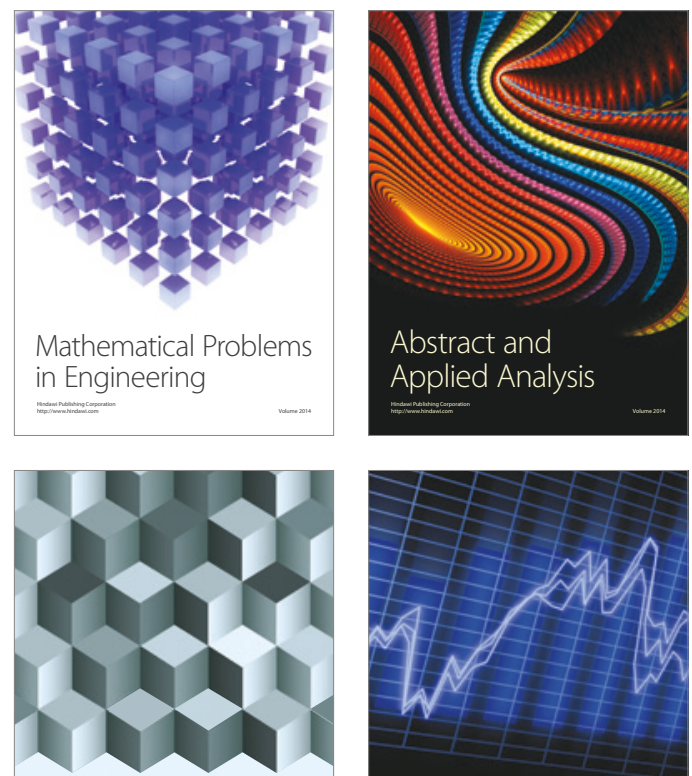

Journal of

Function Spaces

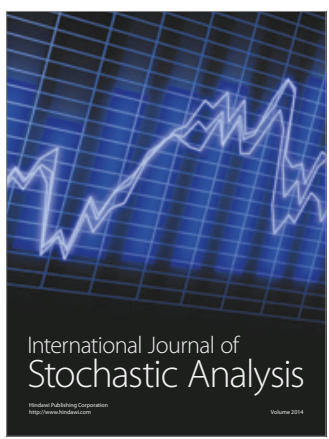

Probability and Statistics
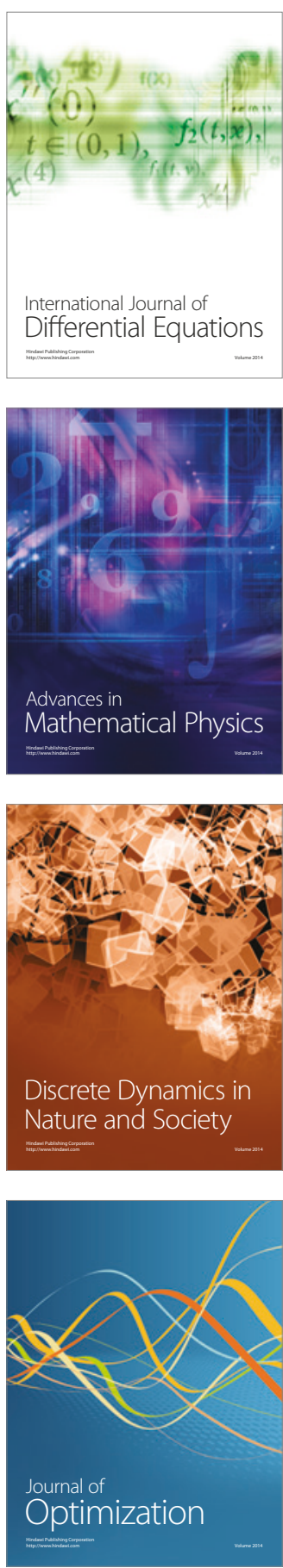